\title{
Very Large Graphs
}

\author{
László Lovász* \\ Dedicated to the memory of Oded Schramm
}

\section{COnTEnts}

1. Introduction 69

1.1. Huge networks 69

1.2. What to ask about them? 70

1.3. How to obtain information about them? 71

1.3.1. Local sampling $\quad 71$

$\begin{array}{ll}\text { 1.3.2. } & \text { Observing global processes } \\ & 72\end{array}$

$\begin{array}{ll}\text { 1.3.3. Left and right homomorphisms } & 72\end{array}$

$\begin{array}{ll}\text { 1.4. How to model them? } & 73\end{array}$

$\begin{array}{ll}\text { 1.4.1. Random graphs } & 73\end{array}$

1.4.2. Randomly growing graphs $\quad 73$

1.4.3. Quasirandom graphs 74

1.5. How to approximate them? 74

1.5.1. The distance of two graphs $\quad 75$

1.5.2. Approximation by smaller: Regularity Lemma $\quad 75$

1.5.3. Approximation by infinite: convergence and limits 76

1.5.4. Optimization problems for graphs 78

$\begin{array}{ll}\text { 1.6. Mathematical tools } & 79\end{array}$

2. Graph parameters $\quad 79$

2.1. Connection matrices and reflection positivity 79

2.2. Homomorphisms from the left 80

2.2.1. Versions of homomorphism numbers 80

2.2.2. Spectra 81

2.3. Homomorphisms to the right 81

2.3.1. Colorings and independent sets 81

2.3.2. Multicuts 81

2.3.3. Statistical physics 83

2.4. Homomorphisms densities in the sparse case 84

${ }^{*}$ Research sponsored by OTKA Grant No. 67867.

(C)2009 International Press 
2.5. Characterizing homomorphism numbers 85

2.5.1. Reflection positivity and extremal graph theory 85

2.5.2. Finite connection rank 86

2.6. Graph algebras $\quad 86$

3. Graph-like structures on probability spaces 88

3.1. Graphons $\quad 89$

3.1.1. Homomorphisms into graphons and from $\begin{array}{lr}\text { graphons } & 89\end{array}$

3.1.2. $W$-random graphs $\quad 91$

$\begin{array}{ll}\text { 3.2. Graphings } & 91\end{array}$

3.2.1. Measure preserving graphs $\quad 91$

3.2.2. Graphings $\quad 92$

3.2.3. Random countable rooted graphs $\quad 92$

4. The cut-distance of two graphs 93

4.1. Two graphs on the same set of nodes 93

4.2. Two graphs with the same number of nodes 94

4.3. Two arbitrary graphs 94

4.4. Distance of graphons $\quad 95$

5. Szemerédi partitions 96

5.1. $\varepsilon$-regular bipartite graphs and the original lemma 96

5.2. Weak Regularity Lemma and distance of graphs 97

5.3. Strong Regularity Lemma and compactness 98

5.4. Partitions into sets with small diameter $\quad 99$

5.4.1. Small diameter sets and regularity $\quad 99$

5.4.2. Computational applications $\quad 99$

5.5. Regularity Lemmas for bounded degree graphs? 101

6. Convergence and limits I: the dense case 102

6.1. Subgraph sampling 102

6.2. Convergence in distance 102

6.3. Convergence from the right 103

6.4. Sampling and distance 103

6.5. Dense limit 105

6.5.1. Equivalent descriptions of the limit 106

$\begin{array}{ll}\text { 6.5.2. Examples } & 107\end{array}$

6.6. Convergence from the right 109

6.7. Limits of other dense combinatorial structures 109

7. Convergence and limits II: bounded degree graphs 110

$\begin{array}{ll}\text { 7.1. Neighborhood sampling } & 110\end{array}$

7.2. Local (weak) limit 110

7.2.1. Different forms 110

7.2.2. Is the limit informative enough? 111

7.3. Convergence from the right 112

8. Testing 113

8.1. Sample concentration 113

8.2. Parameter estimation 113 
8.3. Dense property testing

9. Extremal graph theory

9.1. Some classical results

9.2. Algebraic proofs of extremal graph results 119

9.3. Positivstellensatz for graphs and spectral norms 121

9.4. The maximum distance from a hereditary graph property 122

9.5. Which graphs are extremal? (Finitely forcible graphons) 122

\section{Introduction}

1.1. Huge networks. In the last decade it became apparent that a large number of the most interesting structures and phenomena of the world can be described by networks: separable elements, with connections (or interactions) between certain pairs of them.

- Among such a networks, the best known and the most studied is the internet. Moreover, the internet (as the physical underlying network) gives rise to many of the networks: the network of hyperlinks (web, logical Internet), Internet based social networks, distributed data bases, etc. The size of the internet is growing fast: currently the number of web pages may be 30 billion or more, and the number of devices is probably more than a billion.

- Social networks are basic objects of many studies in the area of sociology, history, epidemiology and economics. The largest social network is the acquaintance graph of all living people, with about 7 billion nodes.

- Biology contributes ecological networks, networks of interactions between proteins, and the human brain, just to mention a few. The human brain is really large for its mass, having about $10^{11}$ nodes.

- Statistical physics studies the interactions between large numbers of discrete particles, where the underlying structure is often described by a graph. For example, a crystal can be thought of as a graph whose nodes are the atoms and whose edges represent chemical bonds. A perfect crystal is a rather boring graph, but impurities and imperfections create interesting graph-theoretical digressions. 12 gram of a diamond has about $6 \times 10^{23}$ nodes.

- Some of the largest networks in engineering occur in chip design. Even though these networks are man-made and planned, many of their properties are difficult to determine by computation due to their huge size. There can be more than a billion transistors on a chip now. 
- To be pretentious, we can say that the whole universe is a single (really huge, possibly infinite) network, where the nodes are events (interactions between elementary particles), and the edges are the particles themselves. This is a network with perhaps $10^{80}$ nodes.

These huge networks pose exciting challenges for the mathematician. Graph Theory (the mathematical theory of networks) has been one of the fastest developing areas of mathematics in the last decades; with the appearance of the Internet, however, it faces fairly novel, unconventional problems. In traditional graph theoretical problems the whole graph is exactly given, and we are looking for relationships between its parameters or efficient algorithms for computing its parameters. On the other hand, very large networks (like the Internet) are never completely known, in most cases they are not even well defined. Data about them can be collected only by indirect means like random local sampling or by monitoring the behavior of various global processes.

Dense networks (in which a node is adjacent to a positive percent of others nodes) and sparse networks (in which a node has a bounded number of neighbors) show a very diverse behavior. From a practical point of view, sparse networks are more important, but at present we have more complete theoretical results for dense networks.

1.2. What to ask about them? Let us discuss three possible questions that can be asked about a really large graph, say the internet.

Question 1. Does the graph have an odd number of nodes?

This is a very basic property of a graph in the classical setting. For example, it is one of the first theorems or exercises in a graph theory course that every graph with an odd number of nodes has a node with even degree.

But for the internet, this question is clearly nonsense. Not only does the number of nodes change all the time, with devices going online and offline, but even if we fix a specific time like 12:00am today, it is not well-defined: there will be computers just in the process of booting up, breaking down etc.

QUeSTION 2. What is the average degree of nodes?

This, on the other hand, is a meaningful question. Of course, the average degree can only be determined with a certain error, and it will change with technology or the social composition of users; but at a given time, a good approximation can be sought (I am not speaking now about how to find it).

QUESTION 3. Is the graph connected?

To this question, the answer is almost certainly no: somewhere there will be a faulty router with some unhappy users on the wrong side of it. But this is not the interesting way to ask the question: we should consider the internet disconnected if, say, an earthquake combined with a sunflare severs all connections between the Old and New worlds. So we want to ignore 
small components that are negligible with respect to the whole graph, and consider the graph disconnected only if it decomposes into two parts which are commeasurable with the whole. On the other hand, we may want to allow that the two parts be connected by a few edges, and still consider the graph disconnected.

QUESTION 4. Find the largest cut in the graph.

(This means to find the partition of the nodes into two classes so as to maximize the number of edges connecting the two classes.) This example shows that even if the question is meaningful, it is not clear in what form can we expect the answer. The fraction of edges contained in the largest cut can be determined relatively easily (with and error that is small with large probability); but how to specify the largest cut itself (or even an approximate version of it)?

1.3. How to obtain information about them? If we face a large network (think of the internet) the first challenge is to obtain information about it. Often, we don't even know the number of nodes.

1.3.1. Local sampling. Properties of very large graphs can be studied by sampling small subgraphs. The theory of this, called property testing in computer science, emerged in the last decade, and will be one of the main concerns of this paper.

In the case of dense graphs $G$, the sampling process is simple: we select independently a fixed number $k$ of random nodes, and determine the edges between them, to get a random induced subgraph. We'll call this subgraph sampling. For each graph $F$, this defines a probability of seeing $F$ when $|V(F)|$ nodes are sampled, and so it gives a probability distribution $\sigma_{G, k}$ on all graphs with $k$ (labeled) nodes. It turns out that this sample contains enough information to determine many properties and parameters of the graph (with an error that is with large probability arbitrarily small if $k$ is sufficiently large depending only on the error bound).

To get a mathematically exact description of algorithms for very large graphs, we define a subgraph sampling oracle as a black box that, for a given positive integer $m$, returns a random $m$-node graph from some (otherwise unknown) distribution. We think of this as a random induced subgraph of a very large, otherwise unknown graph $G$. We assume that the oracle is consistent in the sense that for any $k$ there is a graph $G$ such that the distribution of the $k$-samples from $G$ is arbitrarily close to the distribution of the answers by the oracle. (Theorem 6.13 will give a characterization of consistent distributions.)

In the case of sparse graphs with bounded degree, the subgraph sampling method gives a trivial result: the sampled subgraph will almost certainly be edgeless. Probably the most natural way to fix this is to consider neighborhood sampling. Let $\mathcal{G}_{d}$ denote the class of finite graphs with all degrees bounded by $d$. For $G \in \mathcal{G}_{d}$, select a random node and explore its 
neighborhood to a given depth $m$. This provides a probability distribution $\rho_{G, m}$ on graphs in $\mathcal{G}_{d}$, with a specified root node, such that all nodes are at distance at most $m$ from the root. We will shortly refer to these rooted graphs as $m$-balls. Note that the number of possible $m$-balls is finite if $d$ and $m$ are fixed. We can formulate this abstractly as a neighborhood sampling oracle, a black box that, for a given positive integer $m$, returns an $m$-ball.

The situation for sparse graphs is, however, less satisfactory than for dense graphs, for two reasons. First, a full characterization of consistent neighborhood sampling oracles is not known (cf. Conjecture 7.2). Second, neighborhood sampling does not reveal important global properties of the graph like expansion. This suggests looking at further possibilities. Suppose, for example, that instead of exploring the neighborhood of a single random node, we could select two (or more) random nodes and determine simple quantities associated with them, like pairwise distances, maximum flow, electrical resistance, hitting times of random walks. What information can be gained by such tests? Is there a "complete" set of tests that would give enough information to determine the global structure of the graph to a reasonable accuracy? These methods should lead to different theories of large graphs and their limit objects, largely unexplored.

Sample distribution (in both the dense and sparse cases) are equivalent to counting induced subgraphs of a given type. Instead of this, we could count homomorphism (or injective homomorphisms) of a "small" graphs into the graph. The connection with sample distribution can be expressed by inclusion-exclusion formulas, and it is not essential. Often homomorphism numbers are algebraically better behaved, and they also have the advantage that they suggest different, "dual" approaches by reversing the arrows in the category of graph homomorphisms.

1.3.2. Observing global processes. Another source of information about a network is the observation of the behavior of various global processes either globally (through measuring some global parameter), or locally (at one node, or a few neighboring nodes, but for a longer time). Statistical physical models on the graph are examples of the first kind of approach (we return to them in Section 2.3.3). Crawlers can be considered as examples of the second, and there are some sporadic results about the local observation of simpler, random processes $[\mathbf{1 4}, \mathbf{1 5}]$. A general theory of such local observation has not emerged yet though.

1.3.3. Left and right homomorphisms. Instead of testing, it is often more convenient to talk about homomorphisms (adjacency-preserving maps) between graphs. This leads to the following setup. If we are given a (large) graph $G$, we may try to study its local structure by counting homomorphisms from various "small" graphs $F$ into $G$; and we can study its global structure by counting its homomorphisms into various small graphs $H$. The first type of information is closely related (in many cases, equivalent) to 
sampling, while the second is related to statistical physics. As in statistical physics, one needs weighted graphs $H$ here to get meaningful results.

\subsection{How to model them?}

1.4.1. Random graphs. We are celebrating the 50-th birthday of random graphs this year: The simplest random graph model was developed by Erdös and Rényi [44] and Gilbert [55] in 1959. Given a positive integer $n$ and a real number $0 \leq p \leq 1$, we generate a random graph $\mathbb{G}(n, p)$ by taking $n$ nodes, say $[n]=\{1, \ldots, n\}$, and connecting any two of them with probability $p$, making an independent decision about every pair.

There are alternate models, essentially equivalent: we could fix the number of edges $m$, and then choose a random $m$-element subset of the set of pairs in $[n]$, uniformly from all such subsets. This random graph $\mathbb{G}(n, m)$ is very similar to $\mathbb{G}(n, p)$ when $m=p\left(\begin{array}{l}n \\ 2\end{array}\right)$. Another model, closer to some of the more recent developments, is evolving random graphs, where edges are added one by one, always choosing uniformly from the set of unconnected pairs. Stopping this process after $m$ steps, we get $\mathbb{G}(n, m)$.

Erdős-Rényi random graphs have many interesting, often surprising properties, and a huge literature, see $[\mathbf{2 0 , 6 8}]$. One conventional wisdom about random graphs with a given edge density is that they are all alike. Their basic parameters, like chromatic number, maximum clique, triangle density, spectra etc. are highly concentrated. This fact will be an important motivation when defining the right measure of global similarity of graphs.

Many generalizations of this random graph model have been studied. For example, one could have different probabilities assigned to different edges. A variation of this idea, discovered independently in [85], [22] and perhaps elsewhere, is the notion of $W$-random graphs, to be discussed in Section 3.1.2 and used throughout these notes.

1.4.2. Randomly growing graphs. Random graph models on a fixed set of nodes, discussed above, fail to reproduce important properties of reallife networks. For example, the degrees of Erdős-Rényi random graphs follow a binomial distribution, and so they are asymptotically normal if the edge probability $p$ is a constant, and asymptotically Poisson if the expected degree is constant (i.e., $p=p(n) \sim c / n$ ). In either case, the degrees are highly concentrated around the mean, while the degrees of real life networks tend to obey the "Zipf phenomenon", which means that the tail of the distribution decreases according to a power law.

In 2002 Albert and Barabási [1, 13] created a random network model growing according to natural rules, which could reproduce this behavior. Since then a lot of variations of growing networks were introduced. The process of graph generation usually consists of random steps obeying some local rules.

This is perhaps the first point which suggests one of our main tools, namely assigning limits to sequences of graphs. Just as the Law of Large Numbers tells us that adding up more and more independent random 
variables we get an increasingly deterministically behaving number, these growing graph sequences tend to have a well-defined structure, independent of the random choices made along the way. In the limit, the randomness disappears, and the asymptotic behavior of the sequence can be described by a well-defined limit object. You will find more on this in Sections 1.5.3 and 6.5 .

1.4.3. Quasirandom graphs. The theory of quasirandom graphs, introduced by Thomason [117] and Chung, Graham and Wilson [33], is based on the following observation: not only have random graphs a variety of quite strict properties (with large probability), but for several of these basic properties, the exceptional graphs are the same. In other words, any of these properties implies the others, regardless of any stochastic consideration.

To make this idea precise, we consider a sequence of graphs $\left(G_{n}\right)$ with $\left|V\left(G_{n}\right)\right| \rightarrow \infty$. For simplicity, assume that $\left|V\left(G_{n}\right)\right|=n$. Let $0<p<1$ be a real number. Consider the following properties of these graphs.

(P1) All degrees are asymptotically $p n$ and all codegrees (numbers of common neighbors of two nodes) are asymptotically $p^{2} n$.

(P2) For every fixed graph $F$, the number of homomorphisms of $F$ into $G_{n}$ is asymptotically $p^{|E(F)|} n^{|V(F)|}$.

(P3) The number of edges is asymptotically $p n^{2} / 2$ and the number of 4 -cycles is asymptotically $p^{4} n^{4} / 8$.

(P4) The number of edges induced by a set of nodes of size $\alpha n$ is asymptotically $p \alpha^{2} n^{2} / 2$.

All these properties hold with probability 1 if $G_{n}=\mathbb{G}(n, p)$. However, more is true: if a graph sequence satisfies either one of them, then it satisfies all [33]. Such graph sequences are called quasirandom. The four properties above are only a sampler; there are many other random-like properties that are also equivalent to these $[\mathbf{3 3}, \mathbf{1 0 8}, \mathbf{1 0 9}]$.

Many interesting deterministic graph sequences are quasirandom. We mention an important example from number theory:

ExAmple 1.1. Paley graphs. Let $p_{n}$ be the $n$-th prime congruent 1 modulo 4 , and let us define a graph on $\left\{1, \ldots, p_{n}\right\}$ by connecting $i$ and $j$ if and only if $i-j$ is a quadratic residue. The Paley graphs form a quasirandom sequence.

The theory of convergent graph sequences (Section 6) can be considered as a rather far-reaching generalization of quasirandom sequences.

1.5. How to approximate them? We want a compact approximate description of a very large network, usually in the form a (relatively) small networks or at least a network with a compact description. To make this mathematically precise, we need to define what we mean by two graphs to be "similar" or "close", and describe what kind of structures we use for approximation. 
1.5.1. The distance of two graphs. There are many ways of defining the distance of two graphs $G$ and $G^{\prime}$. Suppose that the two graphs have a common node set $[n]$. Then a natural notion of distance is the edit distance, defined as the number of edges to be changed to get from one graph to the other. Since our graphs are very large, we want to normalize this, and define

$$
d_{1}\left(G, G^{\prime}\right)=\frac{\left|E(G) \triangle E\left(G^{\prime}\right)\right|}{\left(\begin{array}{l}
n \\
2
\end{array}\right)} .
$$

While this distance plays an important role in the study of testable graph properties, it does not reflect structural similarity well. To raise one objection, consider two random graphs on $[n]$ with edge-density $1 / 2$. As mentioned in the introduction, these graphs are very similar from almost every aspect, but their normalized edit distance is large (about $1 / 2$ with large probability). One might try to improve this by relabeling one of them to get the best overlay minimizing the edit distance; but the improvement would be marginal $(o(1))$.

Another trouble with the notion of edit distance is that it is only defined when the two graphs have the same number of nodes.

We could base the measurement of distance on sampling. We define the sampling distance of two graphs $G$ and $G^{\prime}$ by

$$
d_{\text {sample }}\left(G, G^{\prime}\right)=\sum_{k=1}^{\infty} \frac{1}{2^{k}} d_{\mathrm{tv}}\left(\sigma_{G, k}, \sigma_{G^{\prime}, k}\right)
$$

(where $d_{\mathrm{tv}}(\alpha, \beta)=\sup _{X}|\alpha(X)-\beta(X)|$ denotes the total variation distance of the distributions $\alpha$ and $\beta$ ). Here the coefficients $1 / 2^{k}$ are quite arbitrary, only to make the sum convergent. This distance, however, would not directly reflect any structural similarity.

In Section 4 we will define a further distance between graphs, which will be satisfactory from all these points of view: it will be defined for two graphs with possibly different number of nodes, the distance of two random graphs with the same edge density will be very small, and it will reflect global structural similarity. It will define the same topology as $d_{\text {sample }}$.

The construction of the sampling distance can be carried over to bounded degree graphs, by replacing in (1) the sampling distributions $\sigma_{G, k}$ by the neighborhood distributions $\rho_{G, k}$. We must point out, however, that it seems to be difficult to define a notion of distance between two graphs with bounded degree reflecting global similarity.

1.5.2. Approximation by smaller: Regularity Lemma. As the exact description of huge networks is not known, and they are too big for direct study (e.g., for testing different algorithms or protocols directly on the whole internet), an important operation would be to "scale down" by producing a smaller network with similar properties. The main tool for doing so is the "Szemerédi-partition" or "Regularity Lemma". Szemerédi developed 
his Regularity Lemma for his celebrated proof of the Erdős-Turán Conjecture on arithmetic progressions in dense sets of integers in 1975. Since then, the Lemma has emerged as a fundamental tool in graph theory, with many applications in extremal graph theory, combinatorial number theory, graph property testing etc., and became a true focus of research in the past years.

This lemma can be viewed as an archetypal example of dichotomy between randomness and structure, where we try to decompose a (large and complicated) object $A$ into a more highly structured object $A^{\prime}$ with a (quasi)random perturbation (cf. Tao [116]). The highly structured part may be easier to handle, the quasirandom part will often be simpler due to Laws of Large Numbers. We'll introduce this partition in Section 5 (and use it throughout).

Finding the Szemerédi partition of a huge dense graph is an example of the problem posed in Question 4 in Section 1.2. Algorithm 5.4.2 will be an example of a possible solution: how a partition of the nodes can be determined in an implicit form, even if describing for each node which class it belongs to would take too much space.

1.5.3. Approximation by infinite: convergence and limits. This idea can be motivated by how we look at a large piece of metal. This is a crystal, that is a really large graph consisting of atoms and bonds between them. But from many points of view (e.g., the use of the metal in building a bridge), it is more useful to consider it as a continuum with a few important parameters (density, elasticity etc.). Its behavior is governed by differential equations. Can we consider a more general very large graph as some kind of continuum?

One way to make this intuition precise is to consider a growing sequence $\left(G_{n}\right)$ of graphs whose number of nodes tends to infinity, and to define when such a sequence is convergent. (We have mentioned this idea in connection with randomly growing graphs, but now we don't assume anything about how the graphs in the sequence are obtained.) Our discussion of sampling suggests a general principle leading to a definition: we consider samples of a fixed size $k$ from $G_{n}$, and their distribution. We say that the sequence is locally convergent (with respect to the given sampling method) if this distribution tends to a limit as $n \rightarrow \infty$ for every fixed $k$. The family of limiting distributions (one for each $k$ ) can be considered as a limit object of the sequence.

For dense graphs, this notion of convergence was suggested by Erdős, Lovász and Spencer [43], and elaborated by Borgs, Chayes, Lovász, Sós, Szegedy and Vesztergombi $[\mathbf{2 8}, \mathbf{2 9}, \mathbf{3 0}]$. For sparse graphs, this kind of convergence was introduced by Aldous [2] and by Benjamini and Schramm [16]. These notions will be discussed in Sections 6.1 and 7.1, respectively.

The definition above represents the limit of a graph sequence as a collection of probability distributions on graphs, one for each sample size. This is not always a helpful representation of the limit object, and a more explicit description is desirable. A next step is to represent the family of distributions on finite graphs (the samples) by a single probability distribution on 
countable graphs. For sparse graphs, Benjamini and Schramm provide such a description as certain measures on countable rooted graphs with bounded degree (see Section 3.2), and a similar description for dense graph limits is also known as certain ergodic measures on countable graphs $([\mathbf{1 1 1}, \mathbf{9 1}]$; see Theorem 6.13).

More explicit descriptions of these limit objects can also be given. Let us start with the dense case. Here the limit object can be described as a twovariable measurable function $W:[0,1]^{2} \rightarrow[0,1]$, called a graphon (Lovász and Szegedy [85]; see Section 3.1). These limit objects can be considered as weighted graphs with a continuum underlying set, or (if you wish) as graphs on a nonstandard model of the unit interval.

Let us describe an example here; more to follow in Section 6.5.2. The picture on the left hand side of Figure 1 is the adjacency matrix of a graph $G$ with 100 nodes, where the 1's are represented by black squares and the 0 's, by white squares. The graph itself is constructed by a simple randomized growing rule: Starting with a single node, we either add a new node or a new edge; a new node is born with probability $1 / n$, where $n$ is the current number of nodes. (A closely related graph sequence (randomly grown uniform attachment graphs) will be discussed in detail in Section 6.5.2.)

The picture on the right hand side is a grayscale image of the function $U(x, y)=1-\max (x, y)$. The similarity with the picture on the left is apparent; and suggests that the limit of the graph sequence on the left is this function. This turns out to be the case in a well defined sense. It follows that to approximately compute various parameters of the graph on the left hand side, we can compute related parameters of the function on the right hand side. For example, the triangle density of the graph on the left tends (as $n \rightarrow \infty$ ) to the integral

$$
\int_{[0,1]^{3}} U(x, y) U(y, z) U(z, x) d x d y d z .
$$

Two more remarks on the dense case. Of course, a graphon can be infinitely complicated. But in many cases limits of growing graph sequences have a limit graphon that is a continuous function described by a simple
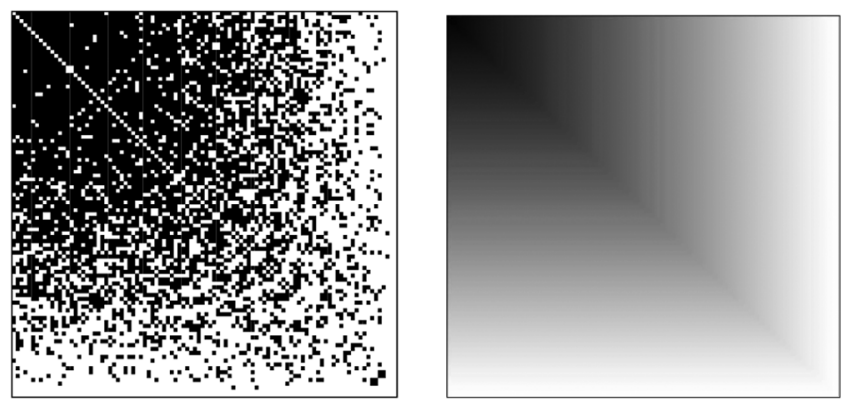

FiguRE 1. A randomly grown uniform attachment graph with 100 nodes 
formula (see a couple of examples in Section 6.5.2). Such a limit graphon provides a very useful approximation of a large dense graph.

Instead of the interval $[0,1]$, we can consider any probability space $(\Omega, \mathcal{A}, \pi)$ with a symmetric measurable function $W: \Omega \times \Omega \rightarrow[0,1]$. This would not give a greater generality, but it is sometimes useful to represent the limit object by other probability spaces. We'll see an example of this in Section 6.5.2.

In the sparse case, the limit object can be described as a graphing (known from group theory or ergodic theory, Elek [37]), or as a measure preserving graph (see Section 3.2), or as a distribution on rooted countable graphs with special properties.

Instead of sampling, we can use dual (global) measurements, more precisely, homomorphisms into fixed small graphs, to define convergence. The remarkable fact is that under the right conditions, this leads to an equivalent notion! (See Sections 6.6, 7.3.)

1.5.4. Optimization problems for graphs. We have presented the theory of convergent graph sequences and their limits as an answer to problems coming from very large networks, but a very strong motivation comes from extremal graph theory.

Consider the following two optimization problems.

Classical optimization problem. Find the minimum of $x^{3}-6 x$ where $x$ is a nonnegative real number.

Graph optimization problem. Find the minimum of $t\left(C_{4}, G\right)$ over all graphs $G$ with $t\left(K_{2}, G\right) \geq 1 / 2$. (Here $t(F, G)$, the homomorphism density of $F$ in $G$, denotes the probability that a random map of $V(F)$ into $V(G)$ preserves the edges. $C_{4}$ denotes the 4 -cycle and $K_{2}$ is the complete graph with 2 nodes.)

The solution of the classical optimization problem is of course $x=\sqrt{2}$. This means that it has no solution over the rationals, but we can find rational numbers that are arbitrarily close to being optimal. If we want a single solution, we have to go to the completion of the rationals, i.e., to the reals.

The graph optimization problem may take a bit more effort to solve, but it is not hard to show that if the edge-density is $1 / 2$, then the 4 -cycle density is larger than $1 / 16$. Furthermore, this density gets arbitrarily close to $1 / 16$ for appropriate families of graphs: the most important example is a random graph with edge-density $1 / 2$ (cf. also Section 1.4.3 and Theorem 9.5).

This suggests that we could try to enlarge the set of (finite) graphs with new objects so that the appropriate extension of our optimization problem has a solution among the new objects. Furthermore, we want that these new objects should be approximable by graphs, just like real numbers are approximable by rationals.

Many of the basic tools in the theory of very large graphs have been first applied in extremal graph theory: the Regularity Lemma [113], convergent graph sequences $[\mathbf{4 3}]$, quasirandom graphs $[\mathbf{1 1 7}, \mathbf{3 3}]$. 
The example above shows that limit objects may provide cleaner formulations of extremal graph theory results, with no error terms. In some cases this goes further, and the limit objects provide a way to state, in an exact way, questions like "How do extremal graphs look like?". They have similar uses in the theory of computing. We discuss these applications in Sections 8 and 9.

1.6. Mathematical tools. It is clear from the above that this area is at the crossroads of different fields of mathematics. Graph theory and computer science are the main sources, and probability and mathematical statistics are crucial tools. Group theory, in particular finitely generated groups, have provided many of the questions and ideas in the theory of limits of graphs with bounded degree. Ergodic theory may play a similar role in the dense case. Measure theory is needed, and an important new general proof method uses nonstandard analysis.

We will discuss one further tool, namely Frobenius algebras, which are used in the proofs of characterization theorems of homomorphism functions, but also in some other studies of graph parameters; see Section 2.6.

\section{Graph parameters}

A graph parameter is a real valued function defined on isomorphism types of graphs (including the graph $K_{0}$ with no nodes and edges). A simple graph parameter is defined only on isomorphism types of simple graphs (i.e., on graphs with no loops or multiple edges). A graph parameter $f$ is multiplicative if $f(G)=f\left(G_{1}\right) f\left(G_{2}\right)$ whenever $G$ is the disjoint union of $G_{1}$ and $G_{2}$. We say that a graph parameter is normalized if its value on $K_{1}$, the graph with one node and no edge, is 1 . Note that if a graph parameter is multiplicative and not identically 0 , then its value on $K_{0}$ (the graph with no nodes and no edges) is 1 .

2.1. Connection matrices and reflection positivity. A $k$-labeled graph is a graph in which $k$ of the nodes are labeled by $1, \ldots, k$ (there may be any number of unlabeled nodes). A 0-labeled graph is just an unlabeled graph.

Let $F_{1}$ and $F_{2}$ be two $k$-labeled graphs. We define the $k$-labeled graph $F_{1} F_{2}$ by taking their disjoint union, and then identifying nodes with the same label. Clearly this multiplication is associative and commutative. For two 0-labeled graphs, $F_{1} F_{2}$ is their disjoint union.

Let $f$ be any graph parameter and fix an integer $k \geq 0$. We define the $k$-th connection matrix of the graph parameter $f$ as the (infinite) symmetric matrix $M(f, k)$, whose rows and columns are indexed by (isomorphism types of) $k$-labeled graphs, and the entry in the intersection of the row corresponding to $F_{1}$ and the column corresponding to $F_{2}$ is $f\left(F_{1} F_{2}\right)$.

We call the graph parameter reflection positive if all the corresponding connection matrices are positive semidefinite. 


\subsection{Homomorphisms from the left}

2.2.1. Versions of homomorphism numbers. For two finite graphs $F$ and $G$, let $\operatorname{hom}(F, G)$ denote the number of homomorphisms of $F$ into $G$ (adjacency-preserving maps from $V(F)$ to $V(G)), \operatorname{inj}(F, G)$, the number of injective homomorphisms of $F$ into $G$, and ind $(F, G)$, the number of embedding of $F$ into $G$ as an induced subgraph.

These quantities are closely related:

$$
\operatorname{inj}(F, G)=\sum_{F^{\prime} \supseteq F} \operatorname{ind}\left(F^{\prime}, G\right),
$$

where $F^{\prime}$ ranges over all graphs obtained from $F$ by adding edges, and

$$
\operatorname{hom}(F, G)=\sum_{F^{\prime \prime}} \operatorname{inj}\left(F^{\prime \prime}, G\right),
$$

where $F^{\prime \prime}$ ranges over all graphs obtained from $F$ by identifying nodes. Conversely, ind can be expressed by inj, which in turn can be expressed by hom using inclusion-exclusion.

This definition can be extended to the case when $G$ has nodeweights $\alpha_{v}$ and edgeweights $\beta_{u v}$ :

$$
\operatorname{hom}(F, G)=\sum_{\varphi: V(F) \rightarrow V(G)} \prod_{u \in V(F)} \alpha_{\varphi(u)}(G) \prod_{u v \in E(F)} \beta_{\varphi(u), \varphi(v)}(G) .
$$

We often normalize these homomorphism numbers, and consider the homomorphism densities

$$
t(F, G)=\frac{\operatorname{hom}(F, G)}{|V(G)|^{|V(F)|}},
$$

which is the probability that a random map of $V(F)$ into $V(G)$ is a homomorphism. We can define similarly

$$
t_{\mathrm{inj}}(F, G)=\frac{\operatorname{inj}(F, G)}{n(n-1) \cdots(n-k+1)}
$$

and

$$
t_{\text {ind }}(F, G)=\frac{\operatorname{ind}(F, G)}{n(n-1) \cdots(n-k+1)} .
$$

We have

$$
t_{\text {inj }}(F, G)=\sum_{F^{\prime} \supseteq F} t_{\text {ind }}\left(F^{\prime}, G\right)
$$

and the inversion formula

$$
t_{\text {ind }}(F, G)=\sum_{F^{\prime} \supseteq F}(-1)^{\left|E\left(F^{\prime}\right) \backslash E(F)\right|} t_{\text {inj }}\left(F^{\prime}, G\right) .
$$


For hom and inj the relationship is not so simple due to the different normalization, but recalling that we are interested in large graphs $G$, the following fact is usually enough to go between them:

$$
t_{\text {inj }}(F, G)-t(F, G)=O\left(\frac{1}{|V(G)|}\right) .
$$

We note that $t_{\text {ind }}(F, G)$ is the probability that sampling $V(F)$ nodes of $G$, we see the graph $F$. So it follows that (for very large graphs, up to the error in (6)) subgraph sampling provides the same information as any of the homomorphism densities $t, t_{\text {inj }}, t_{\text {ind }}$.

2.2.2. Spectra. Homomorphisms of "small" graphs into $G$ are related to sampling, as mentioned earlier. There are less obvious applications of these numbers.

Example 2.1. If $C_{k}$ denote the cycle on $k$ nodes, then $\operatorname{hom}\left(C_{k}, G\right)$ is the trace of the $k$-th power of the adjacency matrix of the graph $G$. In other words,

$$
\operatorname{hom}\left(C_{k}, G\right)=\sum_{i=1}^{n} \lambda_{i}^{k}
$$

where $\lambda_{1}, \ldots, \lambda_{n}$ are the eigenvalues of the adjacency matrix of $G$. From here, eigenvalues with large absolute value can be recovered. For example, $\operatorname{hom}\left(C_{2 k}, G\right)^{1 /(2 k)}$ tends to the largest eigenvalue of $G$ as $k \rightarrow \infty$.

\subsection{Homomorphisms to the right}

2.3.1. Colorings and independent sets. Several important graph parameters can be expressed in terms of homomorphisms into fixed "small" graphs.

ExAmple 2.2. If $K_{q}$ denotes the complete graph with $q$ nodes (no loops), then $\operatorname{hom}\left(G, K_{q}\right)$ is the number of colorings of the graph $G$ with $q$ colors, satisfying the usual condition that adjacent nodes must get different colors.

ExAmple 2.3. Let $H$ be obtained from $K_{2}$ by adding a loop at one of the nodes. Then $\operatorname{hom}(G, H)$ is the number of independent sets of nodes in $G$.

2.3.2. Multicuts. An important graph parameter is the maximum cut $\operatorname{Maxcut}(G)$, the maximum number of edges between a set $S \subseteq V(G)$ of nodes and its complement. While finding minimum cuts is perhaps more natural, the maximum cut problem comes up when we want to approximate general graphs by bipartite graphs, in computing ground states in statistical physics (see next section), and in many other applications. For our purposes, it will be more convenient to consider the normalized maximum cut, defined by

$$
\operatorname{maxcut}(G)=\frac{\operatorname{Maxcut}(G))}{|V|^{2}}=\max _{S \subseteq V} \frac{e_{G}(S, V \backslash S)}{|V|^{2}}
$$

(here $e_{G}(X, Y)$ denotes the number of edges in $G$ connecting node sets $X$ and $Y)$. 
The following easy fact relates maximum cuts and homomorphism numbers. Let $H$ be the weighted graph on $\{1,2\}$ with nodeweights and edgeweights 1 except for the non-loop edge, which has weight 2 . Then we have the trivial inequalities

$$
2^{\operatorname{Maxcut}(G)} \leq \operatorname{hom}(G, H) \leq 2^{|V(G)|} 2^{\operatorname{Maxcut}(G)},
$$

which upon taking the logarithm and dividing by $|V(G)|^{2}$ becomes

$$
\operatorname{maxcut}(G) \leq \frac{\log _{2} \operatorname{hom}(G, H)}{|V(G)|^{2}} \leq \operatorname{maxcut}(G)+\frac{1}{|V(G)|} .
$$

So the homomorphism number into this simple 2-node graph determines $\operatorname{maxcut}(G)$ asymptotically.

An important extension of the maximum cut problem involves partitions into $q \geq 1$ classes instead of 2 . Instead of just counting edges between different classes, we specify in advance numbers $\beta_{i j}(i, j \in[q])$ such that $\beta_{i j}=\beta_{j i}$. We define the maximum multicut density (with the target weights $\beta_{i j}$ ) as

$$
\operatorname{mmcut}(G, \beta)=\max \frac{1}{|V(G)|^{2}} \sum_{i, j} \beta_{i j} e_{G}\left(S_{i}, S_{j}\right),
$$

where the maximum is taken over all partitions $\left\{S_{1}, \ldots, S_{q}\right\}$ of $V(G)$.

A further important extension is to fix the proportion into which the cut separates the node set. For example, the "maximum bisection problem" asks for the maximum size of a cut that separates the nodes into two equal parts (we allow a difference of 1 if the number of nodes is even). More precisely, we can formulate the restricted multicut problem as follows. We specify (in addition to the $\beta_{i j}$ ) numbers $\alpha_{1}, \ldots, \alpha_{q}>0$ with $\alpha_{1}+\cdots+\alpha_{q}=1$. It is convenient to consider the parameters $\alpha_{i}$ and $\beta_{i j}$ as the nodeweights and edge weights of a weighted graph $H$ with $V(H)=[q]$. Then we are interested in

$$
\mathcal{E}(G, H)=\max \frac{1}{|V(G)|^{2}} \sum_{i, j} \beta_{i j} e_{G}\left(S_{i}, S_{j}\right),
$$

where $\left\{S_{1}, \ldots, S_{q}\right\}$ ranges over all partitions of $V(G)$ such that

$$
|| S_{i}\left|-\alpha_{i}\right| V(G)||<1 \quad(i=1, \ldots, q) .
$$

(This can be defined for all graphs $H$ with positive nodeweights, by scaling the nodeweights so that they sum to 1.)

The following extension of (7) is easy to prove: for $H$ fixed and $|V(G)| \rightarrow \infty$

$$
\frac{\log _{2} \operatorname{hom}(G, H)}{|V(G)|^{2}}=\operatorname{mmcut}(G, \beta)+O\left(\frac{1}{|V(G)|}\right) .
$$

(Note that $\log _{2} \operatorname{hom}(G, H) /|V(G)|^{2}$ is asymptotically independent of the node weights of $H$.)

The restricted maximum multicut problem is also related to counting homomorphisms, but the relationship is a little more complicated. Let $G$ be a (very large) simple graph and $H$, a weighted graph with $\mathrm{V}(\mathrm{H})=[\mathrm{q}]$. 
In the definition of $t(G, H)$ we considered random maps $V(G) \rightarrow V(H)$, where the image of each node is chosen independently from the distribution on $V(H)$ defined by the node weights. For most of these random maps $\varphi$, $\left|\varphi^{-1}(i)\right| \approx \alpha_{i}(H)|V(G)| \mid$ by the law of large numbers. It turns out that often it is advantageous to restrict ourselves to maps that are "typical" in this sense. More precisely, let $S(G, H)$ denote the set of those maps $\varphi: V(G) \rightarrow V(H)$ for which ||$\varphi^{-1}(i)\left|-\alpha_{i}\right| V(G)||<1$ for all $i \in V(H)$. Using this notation, we can write

$$
\operatorname{rmcut}(G, H)=\max _{\varphi \in S(G, H)} \sum_{u, v \in V(G)} \beta_{\varphi(u), \varphi(v)} .
$$

Let $\widetilde{H}$ be the weighted graph in which the edge weights are $\widetilde{\beta}_{i j}=\exp \left(\beta_{i j}\right)$ instead of $\beta_{i j}$. If we define

$$
\operatorname{hom}^{*}(G, \widetilde{H})=\sum_{\varphi \in S(G, H)} \prod_{u v \in E(G)} \widetilde{\beta}_{\varphi(u), \varphi(v)},
$$

then the following inequality analogous to (10) holds for $|V(G)| \rightarrow \infty$ :

$$
\operatorname{rmcut}(G, H)=\frac{\log \operatorname{hom}^{*}(G, \widetilde{H})}{|V(G)|^{2}}+O\left(\frac{1}{|V(G)|}\right) .
$$

2.3.3. Statistical physics. Graph homomorphism functions can be used to express partition functions of various statistical physical models. Two basic types of such models are "hard-core" and "soft-core".

To describe an example of a hard-core model, let $G$ be an $n \times n$ grid, and suppose that every node of $G$ (every "site") can be in one of two states, "UP" or "DOWN". The properties of the system are such that no two adjacent sites can be "UP". A "configuration" is a valid assignment of states to each node. The number of configurations is the number of independent sets of nodes in $G$, which in turn can be expressed as the number of homomorphisms of $G$ into the graph $H$ consisting of two nodes, "UP" and "DOWN", connected by an edge, and with an additional loop at "DOWN".

In a soft-core spin model the sites are again nodes of a graph $G$, which can be in one of $q$ possible states. For any two states $i$ and $j$, we specify an "energy of interaction" in the form of a real number $J_{i j}$. A given configuration (assignment of states) is given by a map $\varphi: V(G) \rightarrow[q]$, and its "energy density" is expressed as

$$
\mathcal{E}_{\varphi}=\frac{2}{|V(G)|^{2}} \sum_{u v \in E(G)} J_{\varphi(u), \varphi(v)},
$$

From this, one defines the partition function as

$$
Z(G, J)=\sum_{\varphi: V(G) \rightarrow[q]} \exp \left(-\mathcal{E}_{\varphi}\right) .
$$

Another important quantity is the ground state energy

$$
\mathcal{E}(G, J)=\min _{\varphi: V(G) \rightarrow[q]} \mathcal{E}_{\varphi} .
$$


Note that both of these quantities are familiar: if we take $\beta=-J$, then $\mathcal{E}(G, J)=-\operatorname{rmcut}(G, \beta)$, and if we take $\beta_{i j}=\exp \left(J_{i j}\right)$, then $Z(G, J)=$ $\operatorname{hom}(G, \beta)$. Even restricted multiway cuts correspond to a quantity studied in statistical physics: it is called microcanonical ground state energy there.

The above definitions don't work well for dense graphs $G$ : as remarked after (10), the numbers $\log _{2} \operatorname{hom}(G, H) /|V(G)|^{2}$ are essentially independent of the node weights of $H$, so we loose information here. In the mean-field theory, we define the mean field partition function of a simple graph $G$ by

$$
Z(G, J)=\sum_{\varphi: V(G) \rightarrow[q]} e^{-|V(G)| \mathcal{E}_{\varphi}} .
$$

The free energy is defined by

$$
\mathcal{F}(G, H)=-\frac{\ln Z(G, H)}{|V(G)|} .
$$

Note that the normalization is different from (13) in the exponent and therefore we only divide by $|V(G)|$ (as opposed to (10)).

For more about this connection, we refer to $[\mathbf{3 0}]$.

2.4. Homomorphisms densities in the sparse case. The best analogue for sparse graphs of the homomorphism density $t(F, G)$ is

$$
s(F, G)=\frac{\operatorname{hom}(F, G)}{|V(G)|},
$$

which we consider for connected graphs $F$. We can interpret this number as follows. For $u \in V(F)$ and $v \in V(G)$, let $\operatorname{hom}_{u \rightarrow v}(F, G)$ denote the number of homomorphisms $\varphi$ of $F$ into $G$ with $\varphi(u)=v$. Now we fix any node $u$ of $F$ and select a uniform random node $v$ of $G$. Then $s(F, G)$ is the expectation of $\operatorname{hom}_{u \rightarrow v}(F, G)$. We can interpret

$$
s_{\text {inj }}(F, G)=\frac{\operatorname{inj}(F, G)}{|V(G)|}, \quad s_{\text {ind }}(F, G)=\frac{\operatorname{ind}(F, G)}{|V(G)|}
$$

similarly.

REMARK 2.4. For bounded degree graphs the order of magnitude of $\operatorname{hom}(F, G)$ (where $F$ is fixed and $V(G)$ tends to infinity) is $|V(G)|^{c(F)}$, where $c(F)$ is the number of connected components of $F$. But since $\operatorname{hom}(F, G)$ is multiplicative over the connected components of $F$, we don't loose any information if we restrict the definition $s(F, G)$ to connected graphs $F$.

The sparse homomorphism densities (17) contain the same information as the distribution of neighborhood samples. The proof of this is a bit trickier here than in the dense case.

From the interpretation of $s(F, G)$ given above, we see that it can be obtained as the expectation of the number of $\operatorname{hom}_{u \rightarrow v} s(F, \mathbf{B})$, where $\mathbf{B}$ is a random ball from the neighborhood sample distribution $\rho_{G, r}$, with center $v$ and radius $r=|V(F)|$. 
To compute the neighborhood sample distributions from the quantities $s(F, G)$, we first express the quantities $s_{\text {inj }}(F, G)$ via inclusion-exclusion. By a similar argument, we can express the quantities $s_{\text {ind }}(F, G)$.

Next, we consider graphs $F$ together with maps $\delta: V(F) \rightarrow\{0, \ldots, d\}$, and we determine the numbers

$$
s_{\text {ind }}(F, \delta, G)=\frac{\operatorname{ind}(F, \delta, G)}{|V(G)|},
$$

where $\operatorname{ind}(F, \delta, G)$ is the number injections $\varphi: V(F) \rightarrow V(G)$ which embed $F$ in $G$ as an induced subgraph, so that the degree of $\varphi(v)$ is $\delta(v)$. This is again done by an inclusion-exclusion argument.

Given a ball $B$ of radius $r$, the fraction of nodes $v \in V(G)$ for which $B(v, r)=B$ is $\sum_{\delta} \operatorname{ind}(B, \delta, G)$, where the summation extends over all functions $\delta$ which assigns to each node of $B$ at distance $<r$ from the root its degree in $B$. This proves that homomorphism densities and neighborhood sampling are equivalent.

2.5. Characterizing homomorphism numbers. Multigraph parameters of the form hom $(\cdot, H)$, where $H$ is a weighted graph, were characterized by Freedman, Lovász and Schrijver [51].

THEOREM 2.5. Let $f$ be a graph parameter defined on multigraphs without loops. Then $f$ is equal to hom(., $H)$ for some weighted graph $H$ on $q$ nodes if and only if it is reflection positive and $\operatorname{rk}(M(f, k)) \leq q^{k}$ for all $k$.

Several improvements and versions of this result have been obtained. It is shown in $[\mathbf{8 9}]$ that it is enough to assume the rank condition for $k \leq 2$. Analogous characterizations can be given for graph parameters of the form $\operatorname{hom}(\cdot, H)$ where the nodeweights in $H$ are all $1[\mathbf{1 0 6}]$, and where $H$ is an unweighted graph without multiple edges (but with loops allowed) [81]. There is also an analogous (dual) characterization of graph parameters of the form $\operatorname{hom}(F,$.$) , defined on simple graph with loops, where F$ is also a simple graph with loops $[\mathbf{8 1}]$. These results can be extended to directed graphs, hypergraphs, semigroups, and indeed, to all categories satisfying reasonable conditions $[\mathbf{8 2}]$.

The two conditions on connection matrices in the theorem have interesting uses of their own.

2.5.1. Reflection positivity and extremal graph theory. Theorem 6.13 will give a number of equivalent (cryptographic) descriptions of limit objects of growing graph sequences, and it can be used to characterize all reflection positive graph parameters, see Corollary 6.14.

Reflection positivity implies a number of very useful relations between the densities of various subgraphs in a given graph, which in turn can be used to prove results in extremal graph theory. We will illustrate this in Section 9.

We'll return to applications of reflection positivity of connection matrices in the context of continuous generalizations of graphs (Section 9) and in extremal graph theory (Section 9). 
2.5.2. Finite connection rank. The finiteness of the rank of connection matrices is also interesting. One reason to be interested in this question is the fact that such a graph parameter can be evaluated in polynomial time for graphs with bounded treewidth [78].

There are several examples of graph parameters with finite connection rank [77]: the number of perfect matchings, the number of all matchings, the number of Hamiltonian cycles, any evaluation of the Tutte polynomial.

A challenging problem is to determine all graph parameters for which all the connection matrices have finite rank. Homomorphism functions hom $(., H)$ are examples for every weighted graph $H$ (here the nodeweights and edgeweights can be negative). Dual homomorphism densities hom $(F,$. also have finite connection rank. Every evaluation of the Tutte polynomial is a further example.

Very recently Godlin and Makowski proved that all graph parameters which are evaluations of graph polynomials definable in Monadic Second Order Logic have finite connection rank. This result can be used mostly as a tool to prove that certain properties are not definable this way.

Further variants of this problem ask for the characterization of graph parameters with exponentially bounded connection rank, or polynomially bounded connection rank.

2.6. Graph algebras. A quantum graph is defined as a formal linear combination of a finite number of graphs with real coefficients. For every quantum graph $x$, let $N(x)$ be the maximum number of nodes in the graphs occurring in $x$ with nonzero coefficient. The definition of $\operatorname{hom}(F, G)$ and $t(F, G)$ extends to quantum graphs linearly: if $f=\sum_{i=1}^{n} \lambda_{i} F_{i}$ and $g=$ $\sum_{j=1}^{m} \mu_{j} G_{j}$, then we define

$$
\operatorname{hom}(f, g)=\sum_{i=1}^{n} \sum_{j=1}^{m} \lambda_{i} \mu_{j} \operatorname{hom}\left(F_{i}, G_{j}\right) .
$$

Quantum graphs are useful in expressing various combinatorial situations. For example, for any graph $F$ we define

$$
\widehat{F}=\sum_{\substack{F^{\prime}: V\left(F^{\prime}\right)=V(F) \\ E\left(F^{\prime}\right) \supseteq E(F)}}(-1)^{\left|E\left(F^{\prime}\right)\right|} F^{\prime} .
$$

Then $t(\widehat{F}, G)$ is just the probability that a random map $V(F) \rightarrow V(G)$ preserves adjacency as well as non-adjacency.

Let $f$ be any graph parameter and fix an integer $k \geq 0$. Let $\mathcal{Q}_{k}$ denote the (infinite dimensional) vector space of all $k$-labeled quantum graphs. We can turn $\mathcal{Q}_{k}$ into an algebra by using $F_{1} F_{2}$ introduced above as the product of two generators, and then extending this multiplication to the other elements linearly. Clearly $\mathcal{Q}_{k}$ is associative and commutative. The graph $O_{k}$ on $k$ nodes with no edges is the multiplicative unit in $\mathcal{Q}_{k}$. If all nodes 
of $F$ are labeled, then both $F$ and the quantum graph $\widehat{F}$ introduced above (keeping the node labels) are idempotent: $F^{2}=F$ and $\widehat{F}^{2}=\widehat{F}$.

Every graph parameter $f$ can be extended linearly to quantum graphs, and defines an inner product on $\mathcal{Q}_{k}$ by

$$
\langle x, y\rangle:=f(x y) .
$$

This means that our graph algebra is a Frobenius algebra (see [70]). This inner product has nice properties, for example

$$
\langle x, y z\rangle=\langle x y, z\rangle \text {. }
$$

Let $\mathcal{N}_{k}(f)$ denote the kernel of this inner product, i.e.,

$$
\mathcal{N}_{k}(f):=\left\{x \in \mathcal{Q}_{k}: f(x y)=0 \forall y \in \mathcal{Q}_{k}\right\} .
$$

Then we can define the factor algebra

$$
\mathcal{Q}_{k} / f:=\mathcal{Q}_{k} / \mathcal{N}_{k}(f) .
$$

EXAMPle 2.6. As an example, consider the number $\operatorname{pm}(G)$ of perfect matchings in the graph $G$. It is a basic property of this value that subdividing an edge by two nodes does not change it. This can be expressed as $P_{4}-P_{2} \in \mathcal{N}_{2}(\mathrm{pm})$, where $P_{k}$ denotes the paths with $k$ nodes, of which the two endnodes are labeled.

We can introduce a third "product": the tensor product $G \otimes H$ of a $k$-labeled graph $G$ and an $l$-labeled graph $H$ is defined as the $(k+l)$-labeled graph obtained as the disjoint union of $G$ and $H$, where the labels in $H$ are increased by $k$. If $k=l=0$, then the tensor product is the same as the product in the algebra $\mathcal{Q}_{k}$.

The parameter $f$ is reflection positive if and only if the inner product (19) is positive semidefinite on $\mathcal{Q}_{k}$; equivalently, positive definite on $\mathcal{Q}_{k} / f$, so it turns $\mathcal{Q}_{k} / f$ into a Hilbert space. In fact, the factor algebra $\mathcal{Q}_{k} / f$ is a commutative $*$-algebra, which has both a commutative and associative product and a positive definite inner product, related by $\langle x, y z\rangle=\langle x y, z\rangle$.

The dimension of $\mathcal{Q}_{k} / f$ is the rank of the connection matrix. If this rank is a finite number $m$ and the parameter is reflection positive, it follows that $\mathcal{Q}_{k} / f$ is isomorphic $\mathbb{R}^{m}$ endowed with the coordinate-wise product and the usual inner product.

There are many algebraically interesting connections between these algebras, for example, there is an embedding given by the tensor product

$$
\mathcal{Q}_{k} / f \otimes \mathcal{Q}_{l} / f \hookrightarrow \mathcal{Q}_{k+l} / f
$$

which shows that $\operatorname{dim}\left(\mathcal{Q}_{k} / f\right)$ is a superadditive function of $k$.

This nice algebraic structure can be exploited in various ways $[\mathbf{5 1}, \mathbf{7 8}$, 84, 86]. Let us sketch the proof of Theorem 2.5 in an (easier) special case: when there is no degeneracy in the sense that the embedding in (21) is an isomorphism (this is in fact the generic case, which occurs whenever $f=\operatorname{hom}(., H)$, where $H$ has no "twin" nodes nor any nontrivial automorphism). So we have $\operatorname{dim}\left(\mathcal{Q}_{k} / f\right)=q^{k}$ for all $k$. 
Let $p_{1}, \ldots, p_{q}$ be the basis of $\mathcal{Q}_{1} / f$ consisting of idempotents (corresponding to the standard basis vectors in $\left.\mathbb{R}^{q}\right)$. Define $p_{\varphi}=p_{\varphi(1)} \otimes \cdots \otimes p_{\varphi(k)}$ for all $\varphi:[k] \rightarrow[q]$, then the $k$-labeled quantum graphs $p_{\varphi}$ form a basis of $\mathcal{Q}_{k} / f$ consisting of idempotents.

We can define a weighted complete graph $H$ on $[q]$ as follows: let $\alpha_{i}=$ $f\left(p_{i}\right)$ and define $\beta_{i j}$ by expressing the graph $k_{2}$ (a single edge with both nodes labeled) in the idempotent basis:

$$
k_{2}=\sum_{i, j \in[q]} \beta_{i j}\left(p_{i} \otimes p_{j}\right)
$$

This defines nodeweights $\alpha_{i}$ and edgeweights $\beta_{i j}$ for $H$. The nodeweights are positive, since

$$
\alpha_{i}=f\left(p_{i}\right)=f\left(p_{i}^{2}\right)>0 .
$$

The definition of the $\beta_{i j}$ implies that

$$
k_{2}\left(p_{i} \otimes p_{j}\right)=\beta_{i j}\left(p_{i} \otimes p_{j}\right) .
$$

We claim that the weighted graph $H$ obtained this way satisfies $f(G)=$ $\operatorname{hom}(G, H)$ for every multigraph $G$. Indeed, we may assume that $V(G)=[k]$ and all nodes of $G$ are labeled. Then we can write

$$
G=\prod_{u v \in E(G)} K_{u v}
$$

where $K_{u v}$ consists of $k$ labeled nodes and a single edge connecting $u$ and $v$. Equation (22) implies that

$$
p_{\varphi} K_{u v}=\beta_{\varphi(u) \varphi(v)} p_{\varphi} .
$$

Using (20) repeatedly, we get

$G=\left(\sum_{\varphi:[k] \rightarrow[q]} p_{\varphi}\right) G=\sum_{\varphi:[k] \rightarrow[q]} p_{\varphi} \prod_{u v \in E(G)} K_{u v}=\sum_{\varphi:[k] \rightarrow[q]} \prod_{u v \in E(G)} \beta_{\varphi(u) \varphi(v)} p_{\varphi}$

and so

$$
f(G)=\sum_{\varphi:[k] \rightarrow[q]} \prod_{u v \in E(G)} \beta_{\varphi(u) \varphi(v)} \prod_{u \in V(G)} \alpha_{\varphi(u)}=\operatorname{hom}(G, H) .
$$

\section{Graph-like structures on probability spaces}

The aim of this section is to introduce certain analytic objects, which will serve as limit objects for graph sequences, separately in the dense and sparse case. It is an interesting feature of these structures that they have come up in different studies.

In the dense case, several versions of these objects turn out to be equivalent; graphons are very simple objects (2-variable measurable functions), but they turn out to be equivalent, among others, to exchangeable random variables. 
In the bounded degree case, several related, but non-equivalent notions have been proposed, at least one of which (graphings) is also known from group theory.

3.1. Graphons. Let $\mathcal{W}$ denote the space of all bounded symmetric measurable functions $W:[0,1]^{2} \rightarrow \mathbb{R}$ (i.e., $W(x, y)=W(y, x)$ for all $x, y \in[0,1])$. Let $\mathcal{W}_{0}$ denote the set of all functions $W \in \mathcal{W}$ such that $0 \leq W \leq 1$.

A function $W \in \mathcal{W}$ is called a stepfunction, if there is a partition $S_{1} \cup$ $\cdots \cup S_{k}$ of $[0,1]$ into measurable sets such that $W$ is constant on every product set $S_{i} \times S_{j}$. The number $k$ is the number of steps of $W$.

For every weighted graph $G$, we define a stepfunction $W_{G} \in \mathcal{W}_{0}$ as follows. Let $V(G)=[n]$. Split $[0,1]$ into $n$ intervals $J_{1}, \ldots, J_{n}$ of length $\lambda\left(J_{i}\right)=\alpha_{i} / \alpha_{G}$. For $x \in J_{i}$ and $y \in J_{j}$, let

$$
W_{G}(x, y)=\beta_{i j}(G) .
$$

Let $W \in \mathcal{W}$ and let $\varphi:[0,1] \rightarrow[0,1]$ be a measure preserving map. We can define another function $W^{\varphi}$ by

$$
W^{\varphi}(x, y)=W(\varphi(x), \varphi(y)) .
$$

From the point of view of using these functions as continuous analogues of graphs, the functions $W$ and $W^{\varphi}$ are not essentially different (they are related like two isomorphic graphs in which the nodes are labeled differently). One has to be a little careful though, because measure preserving maps are not necessarily invertible, and so the relationship between $W$ and $W^{\varphi}$ is not symmetric. We call two graphons $W$ and $W^{\prime}$ weakly isomorphic, if there is a third graphon $U$ and measure preserving maps $\varphi, \varphi^{\prime}:[0,1] \rightarrow[0,1]$ such that $W=U^{\varphi}$ and $W^{\prime}=U^{\varphi^{\prime}}$ almost everywhere. It is not hard to show that weak isomorphism is an equivalence relation.

Equivalence classes of functions in $\mathcal{W}_{0}$ under weak isomorphism are called graphons. (Sometimes we call a function $W \in \mathcal{W}_{0}$ a graphon; by analogy with graphs, these functions could be called "labeled graphons".)

3.1.1. Homomorphisms into graphons and from graphons. Counting homomorphism into graphs extends to counting homomorphism into graphons in the following sense: For every $W \in \mathcal{W}$ and simple graph $F=(V, E)$, define

$$
t(F, W)=\int_{[0,1]^{V}} \prod_{i j \in E} W\left(x_{i}, x_{j}\right) \prod_{i \in V} d x_{i}
$$

Then it is easy to verify that for every graph $G$,

$$
t(F, G)=t\left(F, W_{G}\right) .
$$

Of the two modified versions of homomorphism densities (2) and (3), the former has not significance in this context since a random assignment 
$i \mapsto x_{i}\left(i \in V(F), x_{i} \in[0,1]\right.$ is injective with probability 1 . But the induced subgraph density is worth defining, and in fact it can be expressed as

$$
t_{\text {ind }}(F, W)=\int_{[0,1] V} \prod_{i j \in E} W\left(x_{i}, x_{j}\right) \prod_{i j \in\left(\begin{array}{c}
V \\
2
\end{array}\right) \backslash E}\left(1-W\left(x_{i}, x_{j}\right)\right) \prod_{i \in V} d x_{i} .
$$

We have then

$$
t_{\text {ind }}(F, G)=t_{\text {ind }}\left(F, W_{G}\right),
$$

and the inclusion-exclusion formula (5) follows by expanding the parentheses in the integrand (24).

Borgs, Chayes and Lovász [26] proved that the homomorphism densities determine the graphon:

THEOREM 3.1. Two graphons are weakly isomorphic if and only if $t(F, W)=t\left(F, W^{\prime}\right)$ for every simple graph $F$.

A natural idea of the proof of this theorem would be to bring every graphon to a "canonical form", so that weakly isomorphic graphons would have identical canonical forms. In the case of functions in a single variable, a canonical form that works in many situations can be obtained through "monotonization": for every bounded real function on $[0,1]$ there is an unique monotone increasing left-continuous function on $[0,1]$ that has, among others, the same moments. For graphons this does not seem to be doable, but the proof of Theorem 3.1 goes by constructing, for every graphon $W$, a "canonical ensemble": a probability distribution on graphons on the same canonical $\sigma$-algebra and weakly isomorphic to $W$, such that two graphons are isomorphic if and only if their ensembles can be coupled so that corresponding graphons are identical.

Alternate proofs of Theorem 3.1 have been given by Diaconis and Janson [35] using the theory of exchangeable random variables, and by Bollobás and Riordan [24] combining Theorem 6.2 below with measure-theoretic arguments.

There is probably no good way to define homomorphism numbers from graphons into graphs or into other graphons. The parameters related to such homomorphisms that extend naturally to graphons are defined by maximization, like the normalized maximum cut, and more generally, restricted maximum multiway cuts. Let $H$ be a weighted graph with $V(H)=[q]$ and $W$, a graphon. Then we can define

$$
\mathcal{E}(W, H)=\sup _{S_{i}} \sum_{i, j \in V(H)} \beta_{i j} \int_{S_{i} \times S_{j}} W(x, y) d x d y
$$

where $\left\{S_{1}, \ldots, S_{q}\right\}$ ranges over all partitions of $[0,1]$ into measurable sets with $\lambda\left(S_{i}\right)=\alpha_{i}(H)$. This quantity does not exactly extend $\mathcal{E}(G, H)$ as defined in (8), but the error is small: it was proved in [30] that for a fixed 
weighted graph $H$,

$$
\mathcal{E}(G, H)-\mathcal{E}\left(W_{G}, H\right)=O\left(\frac{1}{|V(G)|}\right) \quad(|V(G)| \rightarrow \infty) .
$$

3.1.2. $W$-random graphs. A graphon $W$ gives rise to a way of generating random graphs that are more general than the Erdös-Rényi graphs. This construction was introduced by Lovász and Szegedy [85] and Bollobás, Janson and Riordan [22].

Given a graphon $W$ and an integer $n>0$, we can generate a random graph $\mathbb{G}(n, W)$ on node set $[n]$ as follows: We generate $n$ independent numbers $X_{1}, \ldots, X_{n}$ from the uniform distribution on $[0,1]$, and then connect nodes $i$ and $j$ with probability $W\left(X_{i}, X_{j}\right)$, making an independent decision for distinct pairs $(i, j)$.

As a special case, if $W$ is the identically $p$ function, we get "ordinary" random graphs $\mathbb{G}(n, p)$.

We can extend this construction to generating a countable random graph $\mathbb{G}(W)$ on $\mathbb{N}$ : We generate an infinite sequence $X_{1}, X_{2}, \ldots$ of uniformly distributed random points from $[0,1]$, and (as before) connect nodes $i$ and $j$ with probability $W\left(X_{i}, X_{j}\right)$.

Graphons will come up in several ways in our discussions. In Theorem 6.13 we will collect the many disguises in which they occur.

\subsection{Graphings}

3.2.1. Measure preserving graphs. Let $G$ be a graph with node set $[0,1]$, with all degrees bounded by $d$. We call $G$ measurable, if for every (Lebesgue) measurable set $B$ the neighborhood $N(B)$ in $G$ is also measurable.

For every set $A \subseteq[0,1]$ and $x \in[0,1]$, let $d_{A}(x)$ denote the number of neighbors of $x$ in $B$. One can prove using the measurability of $G$ that $d_{A}(x)$ is a measurable function of $x$. We say that $G$ is measure preserving, if it is measurable and for any two measurable sets $A, B$,

$$
\int_{A} d_{B}(x) d x=\int_{B} d_{A}(x) d x .
$$

Assuming that this relation holds, we can define a measure $\mu$ on the Borel sets of $[0,1]^{2}$ by $\mu(A \times B)=\int_{A} d_{B}(x) d x$. This measure is concentrated on the set of edges (which can be considered as a subset of $[0,1]^{2}$ ). Furthermore, the marginals of $\mu$ are absolutely continuous with respect to the Lebesgue measure, and their Radon-Nikodym derivative is the degree function.

In every measure preserving graph $G$, we can define the density $s(F, G)$ of a graph $F$. Indeed, let us recall that $s(F, G)$ is the expectation of $\operatorname{hom}_{v \rightarrow u}(F, G)$, where $v$ is a fixed node of $F$ and $u$ is a random node of $G$. Since we have a probability distribution on $V(G)$, and $\operatorname{hom}_{v \rightarrow u}(F, G)$ is a bounded measurable function of $u$, this definition carries over verbatim.

Similarly, we can talk about the neighborhood distributions $\rho_{G, m}$ in a measure preserving graph. 
3.2.2. Graphings. Let $A_{1}, \ldots, A_{d}, B_{1}, \ldots, B_{d}$ be measurable subsets of $[0,1]$, and let $\varphi_{i}: A_{i} \rightarrow B_{i}$ be bijective measure preserving maps. The tuple $H=\left([0,1], \varphi_{1}, \ldots, \varphi_{d}\right)$ is called a graphing (see $\left.[\mathbf{5 3}, \mathbf{6 9}]\right)$. From every graphing $H$ we get a directed graph $\vec{G}$ on $[0,1]$ by connecting $x$ and $y$ in $[0,1]$ if there is an $i$ such that $y=\varphi_{i}(x)$. The edges of this digraph are colored with $d$ colors in such a way that each color-class defines a measure preserving bijection between two subsets of $[0,1]$.

Forgetting the orientation and the edge-colors of this digraph, we get a measure preserving graph with degrees bounded by $2 d$. A measure preserving graph with its edges colored and oriented so that each color defines a measure preserving bijection is equivalent to a graphing.

It would be perhaps more natural to assume that the maps $\varphi_{1}, \ldots, \varphi_{d}$ are involutions, in which case we get an undirected graph, and we can extend the $\varphi_{i}$ to measure preserving involutions $[0,1] \rightarrow[0,1]$. It is true that for every graphing there is such an involutive graphing defining the same measure preserving graph; but the number of maps may become much larger.

Every measure preserving graph arises from a graphing:

TheOREM 3.2. Let $G$ be a measure preserving graph with degrees bounded by $d$. Then there is a graphing $H=\left([0,1], \varphi_{1}, \ldots, \varphi_{r}\right)$, where $r \leq d^{2}$, such that the underlying graph is $G$.

One way of looking at a representation of a measure preserving graph as a graphing is that it provides a certificate that the graph is measure preserving. The graphing representing a given measure preserving graph may not be unique.

Theorem 3.2 can be viewed as a measure preserving graph version of Shannon's Theorem, which asserts that the edges of a multigraph with maximum degree $d$ can be colored by $3 d / 2$ colors. (For simple graphs, Vizing's Theorem gives the better bound of $d+1$.) The bound $d^{2}$ is probably not optimal in the measure preserving version either.

We will talk about $s(F, H)$ if $F$ is a (finite) graph and $H$ is a graphing. This will mean simply $s(F, G)$, where $G$ is the underlying measure preserving graph.

We note that both in measure preserving graphs and graphings, we could replace the probability space $[0,1]$ by any other standard probability space, but this would not lead to any gain in generality. However, in some cases the presentation of the measure preserving graph or graphing is more natural on other probability spaces.

3.2.3. Random countable rooted graphs. Measure preserving graphs are also related to certain probability distributions on rooted countable graphs, introduced by Benjamini and Schramm [16].

Let $G$ be a measure preserving graph and choose a uniform random point $x \in[0,1]$. The connected component $G_{x}$ of $G$ containing $x$ is a countable graph with degrees bounded by $d$, and with a "root" node $x$. 
Let $\mathbf{G}_{d}$ denote the set of connected countable graphs with all degrees bounded by $d$, rooted at a node. Let $\mathcal{A}_{d}$ denote the $\sigma$-algebra on $\mathbf{G}_{d}$ generated by subsets obtained by fixing a finite neighborhood of the root. The map $x \mapsto G_{x}$ is measurable as a map $[0,1] \rightarrow\left(G_{d}, \mathcal{A}_{d}\right)$, and thus every measure preserving graph $G$ defines a probability distribution $\pi$ on $\left(\mathbf{G}_{d}, \mathcal{A}_{d}\right)$.

Condition 27 implies the following property of the measure $\pi$. Selecting a rooted graph $G$ from $\pi$ and then selecting a uniform random edge from the root, we get a probability distribution $\pi^{*}$ on the set $\mathbf{G}_{d}^{\prime}$ of rooted graphs in $\mathbf{G}_{d}$ with an edge (the "root edge") from the root also specified. We say that $\pi$ is unimodular, if the map $\mathbf{G}_{d}^{\prime} \rightarrow \mathbf{G}_{d}^{\prime}$ obtained by shifting the root node to the other endnode of the root edge is measure preserving with respect to $\pi$.

The measure on $\mathbf{G}_{d}$ obtained from a measure preserving graph is unimodular. Vice versa, every such measure is obtained from a graphing (and hence from a measure preserving graph; Elek [37]).

\section{The cut-distance of two graphs}

The definition of the distance of two arbitrary graphs is quite involved, and we will approach the problem in steps: starting with two graphs on the same node set, then moving to graphs with the same number of nodes (but unrelated), then moving to the general case.

In this section we consider dense graphs. The definitions are of course valid for all graphs, but they give a distance of $o(1)$ between two graphs with edge-density $o(1)$.

4.1. Two graphs on the same set of nodes. Let $G$ and $G^{\prime}$ be two graphs with a common node set $[n]$. The distance notion discussed here was initiated by Frieze and Kannan [52], and elaborated, e.g., in [29]. For an unweighted graph $G=(V, E)$ and sets $S, T \subseteq V$, let $e_{G}(S, T)$ denote the number of edges in $G$ with one endnode in $S$ and the other in $T$ (the endnodes may also belong to $S \cap T$; so $e_{G}(S, S)$ is twice the number of edges spanned by $S$ ). For two graphs $G$ and $G^{\prime}$ on the same node set $[n]$, we define their cut distance by

$$
d_{\square}\left(G, G^{\prime}\right)=\frac{1}{n^{2}} \max _{S, T \subseteq V(G)}\left|e_{G}(S, T)-e_{G^{\prime}}(S, T)\right| .
$$

Note that we are dividing by $n^{2}$ and not by $|S| \times|T|$, which would look more natural. However, dividing by $|S| \times|T|$ would emphasize small sets too much, and the maximum would be attained when $|S|=|T|=1$. With our definition, the contribution of a pair $S, T$ is at most $|T| \cdot|S| / n^{2}$ (for simple graphs).

It is easy to see that $d_{\square}\left(G, G^{\prime}\right) \leq d_{1}\left(G, G^{\prime}\right)$, and in general the two sides are quite different. For example, if $\mathbb{G}$ and $\mathbb{G}^{\prime}$ are two independent random graphs on $[n]$ with edge probability $1 / 2$, then with large probability $d_{\square}\left(G, G^{\prime}\right)=O(1 / \sqrt{n})$. 
4.2. Two graphs with the same number of nodes. If $G$ and $G^{\prime}$ are unlabeled unweighted graphs on different node sets but of the same cardinality $n$, then we define their distance by

$$
\hat{\delta}_{\square}\left(G, G^{\prime}\right)=\min _{\tilde{G}, \tilde{G}^{\prime}} d_{\square}\left(\tilde{G}, \tilde{G}^{\prime}\right),
$$

where $\tilde{G}$ and $\tilde{G}^{\prime}$ range over all labelings of $G$ and $G^{\prime}$ by $1, \ldots, n$, respectively. (The hat above the $\delta$ indicates that the "ultimate" definition will be somewhat different.)

4.3. Two arbitrary graphs. Let $G=(V, E)$ and $G^{\prime}=\left(V^{\prime}, E^{\prime}\right)$ be two graphs with (say) $V=[n]$ and $V^{\prime}=\left[n^{\prime}\right]$. To define their distance, we need a graph operation: for every graph $G$ and positive integer $m$, let $G(m)$ denote the graph obtained from $G$ by replacing each node of $G$ by $m$ nodes, where two new nodes are connected if and only if their predecessors were.

We can use the distance $\hat{\delta}_{\square}$ to define the distance

$$
\delta_{\square}\left(G, G^{\prime}\right)=\lim _{k \rightarrow \infty} \hat{\delta}_{\square}\left(G\left[k n^{\prime}\right], G^{\prime}[k n]\right) .
$$

(Here $G\left(k n^{\prime}\right)$ and $G^{\prime}(k n)$ have the same number of nodes.)

A more complicated but "finite" definition of the same quantity can be given as follows. A fractional overlay of $G$ and $G^{\prime}$ is a nonnegative $n \times n^{\prime}$ matrix $X$ such that $\sum_{u=1}^{n^{\prime}} X_{i u}=\frac{1}{n}$ and $\sum_{i=1}^{n} X_{i u}=\frac{1}{n^{\prime}}$. If $n=n^{\prime}$ and $\sigma: V \rightarrow V^{\prime}$ is a bijection, then $X_{i u}=\frac{1}{n} \mathbf{1}_{\sigma(i)=u}$ is a fractional overlay (which in this case is an honest-to-good overlay). We denote by $\mathcal{X}\left(G, G^{\prime}\right)$ the set of all fractional overlays.

For a matrix $M$, let $\Sigma(M)$ denote the sum of its entries. Then the distance of the two graphs can be described by the following optimization problem:

$$
\delta_{\square}\left(G, G^{\prime}\right)=\min _{X \in \mathcal{X}\left(G, G^{\prime}\right)} \max _{Y, Z \subseteq V \times V^{\prime}}\left|\sum_{\substack{i u \in Y, j v \in Z \\ i j \in E}} X_{i u} X_{j v}-\sum_{\substack{i u \in Y, j v \in Z \\ u v \in E^{\prime}}} X_{i u} X_{j v}\right| .
$$

To illuminate this definition a little, we can think of a fractional overlay as a coupling of the uniform distribution on $V(G)$ with the uniform distribution on $V\left(G^{\prime}\right)$ : it gives a probability distribution $\chi$ on $V(G) \times V\left(G^{\prime}\right)$ whose marginals are uniform. Select two pairs $(i, u)$ and $(j, v)$ from the distribution $\chi$. Then the first sum in (29) is the probability that " $i u \in Y$ and $j v \in Z$ and $i j \in E$ ", and the second sum is the probability that " $i u \in Y$ and $j v \in Z$ and $u v$ is an edge". Thus (29) expresses some form of correlation between $i j$ being an edge and $u v$ being an edge.

One word of warning: $\delta_{\square}$ is only a pseudometric, not a true metric, because $\delta_{\square}\left(G, G^{\prime}\right)$ may be zero for different graphs $G$ and $G^{\prime}$. This is the case e.g. if $G^{\prime}=G(k)$ for some $k$.

Definition (29) can be extended to weighted graphs, but instead of going through the hairy formulas, we postpone this to the next section. 
We conclude with a problem for which only partial results are available. If $G$ and $G^{\prime}$ have the same number of nodes, then the definition of $\delta_{\square}$ does not give back $\hat{\delta}_{\square}$. It was proved in $[\mathbf{2 9}]$ that

$$
\delta_{\square}\left(G, G^{\prime}\right) \leq \hat{\delta}_{\square}\left(G, G^{\prime}\right) \leq 32 \delta_{\square}(G, G)^{1 / 67} .
$$

This is a rather weak result, its significance being that $\delta_{\square}$ and $\hat{\delta}_{\square}$ define the same Cauchy sequences. Alon (unpublished) proved that

$$
\hat{\delta}_{\square}\left(G, G^{\prime}\right) \leq(1+o(1)) \delta_{\square}(G, G)
$$

if $|V(G)|=\left|V\left(G^{\prime}\right)\right| \rightarrow \infty$. We conjecture:

Conjecture 4.1. For any two graphs $G$ and $G^{\prime}$ on $n$ nodes, $\hat{\delta}_{\square}\left(G, G^{\prime}\right) \leq$ $2 \delta_{\square}\left(G, G^{\prime}\right)$.

An analogous result for the edit distance was proved by Pikhurko [96].

4.4. Distance of graphons. This notion of distance extends to graphons as follows (and it is perhaps more natural in that context). We consider on $\mathcal{W}$ the cut norm

$$
\|W\|_{\square}=\sup _{S, T \subseteq[0,1]}\left|\int_{S \times T} W(x, y) d x d y\right|
$$

where the supremum is taken over all measurable subsets $S$ and $T$. It is sometimes convenient the use the corresponding metric $d_{1}(U, W)=\| U-$ $W\}_{\square}$. We define the cut distance

$$
\delta_{\square}(U, W)=\inf _{\varphi} d_{\square}\left(U, W^{\varphi}\right),
$$

where $\varphi$ ranges over all invertible measure preserving maps from $[0,1] \rightarrow$ $[0,1]$, and $W^{\varphi}(x, y)=W(\varphi(x), \varphi(y))$.

The distance $\delta_{\square}$ of graphons is only a pseudometric, since different graphons can have distance zero. This happens precisely when they are weakly isomorphic.

If $G$ and $G^{\prime}$ are weighted graphs, then we have

$$
\delta_{\square}\left(G, G^{\prime}\right)=\delta_{\square}\left(W_{G}, W_{G^{\prime}}\right) .
$$

This could serve as a more natural (but not combinatorial) definition of the distance of two graphs, and we will use it to define the distance of two weighted graphs. Let $K$ denote the graph with a single node of weight 1 , endowed with a loop with weight $1 / 2$. Then for a random graph $\mathbb{G}=$ $\mathbb{G}(n, 1 / 2)$, we have $\delta_{\square}(\mathbb{G}, K)=O(1 / \sqrt{n})$ with large probability.

Going into all the complications with using the cut norm and then minimizing over measure preserving transformations is justified by the following important fact.

TheOREM 4.2. The pseudometric space $\left(\mathcal{W}_{0}, \delta_{\square}\right)$ is compact. 
The proof depends on Szemerédi partitions, to be discussed in Section 5 .

Convergence in the $\|.\|_{\square}$ norm is stronger than weak-*-convergence. To be more precise, if $\left\|W_{n}-W\right\|_{\square} \rightarrow 0(n \rightarrow \infty)$, then it follows immediately from the definition that

$$
\int_{S \times T} W_{n} \rightarrow \int_{S \times T} W
$$

and hence by standard arguments we get that

$$
\int_{[0,1]^{2}} U \cdot W_{n} \rightarrow \int_{[0,1]^{2}} U \cdot W
$$

for every integrable function $U$. However, weak-*-convergence is not equivalent of convergence in the $\|\cdot\|_{\square}$ norm; a counterexample can be obtained e.g. from Example 6.19 (see [31]).

Similar construction can be applied to other norms, e.g., from the $L_{1^{-}}$norm

$$
\|W\|_{1}=\int_{[0,1]^{2}}|W(x, y)| d x
$$

we get

$$
d_{1}(U, W)=\|U-W\|_{1} \quad \text { and } \quad \delta_{1}(U, W)=\inf _{\varphi} d_{1}\left(U, W^{\varphi}\right) .
$$

\section{Szemerédi partitions}

One of the most important tools in understanding large dense graphs is the Regularity Lemma of Szemerédi $[\mathbf{1 1 2}, \mathbf{1 1 3}]$ and its extensions. This lemma has many interesting connections to other areas of mathematics, including analysis $[\mathbf{8 7}, \mathbf{2 3}]$ and information theory $[\mathbf{1 1 4}]$. It also has weaker (but more effective) and stronger versions. Here we survey as much as we need from this rich theory.

5.1. $\varepsilon$-regular bipartite graphs and the original lemma. For a graph $G=(V, E)$ and for $X, Y \subseteq V$, let $e_{G}(X, Y)$ denote the number of edges with one endnode in $X$ and another in $Y$; edges with both endnodes in $X \cap Y$ are counted twice. We denote by $d_{G}(X, Y)=\frac{e_{G}(X, Y)}{|X| \cdot|Y|}$ the density of edges between $X$ and $Y$. If $X$ and $Y$ are disjoint, we denote by $G[X, Y]$ the bipartite graph on $X \cup Y$ obtained by keeping just those edges of $G$ that connect $X$ and $Y$.

Let $\mathcal{P}=\left\{V_{1}, \ldots, V_{k}\right\}$ be a partition of $V$. We say that $\mathcal{P}$ is an equipartition if $\lfloor|V| / k\rfloor \leq\left|V_{i}\right| \leq\left\lceil\left|V_{i}\right| / k\right\rceil$ for all $1 \leq i \leq k$. We define the weighted graph $G_{\mathcal{P}}$ on $V$ by taking the complete graph and weighting its edge $u v$ by $d_{G}\left(V_{i}, V_{j}\right)$ if $u \in V_{i}$ and $v \in V_{j}$.

The Regularity Lemma says, roughly speaking, that every graph has a partition $\mathcal{P}$ into a "small" number of classes such that $G_{\mathcal{P}}$ is "close" to $G$. There are (non-equivalent) forms of this lemma, depending on how we measure the error. 
Let $G$ be a bipartite graph $G$ with bipartition $\{U, W\}$. On the average, we expect that for $X \subseteq U$ and $Y \subseteq W$,

$$
e_{G}(X, Y) \approx d_{G}(X, Y)|X| \cdot|Y| \text {. }
$$

For two arbitrary subsets of the nodes, $e_{G}(X, Y)$ may be very far from this "expected value", but if $G$ is a random graph, then, however, it will be close; random graphs are very "homogeneous" in this respect. We say that $G$ is $\varepsilon$-regular, if

$$
\left|\frac{e_{G}(X, Y)}{|X| \cdot|Y|}-d\right| \leq \varepsilon
$$

holds for all subsets $X \subseteq U$ and $Y \subseteq W$ such that $|X|>\varepsilon|U|$ and $|Y|>\varepsilon|W|$.

Notice that we could not require condition (35) to hold for small $X$ and $Y$ : for example, if both have one element, then the quotient $e_{G}(X, Y) /(|X|$. $|Y|)$ is either 0 or 1 . However, we could replace it by the condition

$$
\left|e_{G}(X, Y)-d\right| X|\cdot| Y|| \leq \varepsilon|U| \cdot|W|
$$

for all $Y \subseteq U$ and $Y \subseteq W$. Indeed, (35) implies (36) for $|X|>\varepsilon|U|$ and $|Y|>\varepsilon|W|$, while if e.g. $|X| \leq \varepsilon|U|$, then $e_{G}(X, Y) \leq \varepsilon|U| \cdot|W|$ and $d|X| \cdot|Y||\leq \varepsilon| U|\cdot| W \mid$, so (36) holds trivially. Conversely, if (36) holds with $\varepsilon$ replaced by $\varepsilon^{3}$, then

$$
\left|\frac{e_{G}(X, Y)}{|X| \cdot|Y|}-d\right| \leq \frac{\varepsilon^{3}|U| \cdot|W|}{|X| \cdot|Y|}<\varepsilon
$$

if $|X|>\varepsilon|U|$ and $|Y|>\varepsilon|W|$.

With these definitions, the Regularity Lemma can be stated as follows:

Lemma 5.1 (Szemerédi Regularity Lemma, usual form). For every $\varepsilon>0$ there is a $k=k(\varepsilon)$ such that every graph $G=(V, E)$ on at least $k$ nodes has an equipartition $\left\{V_{1}, \ldots, V_{k}\right\}(1 / \varepsilon \leq k \leq k(\varepsilon))$ such that for all but $\varepsilon k^{2}$ pairs of indices $1 \leq i<j \leq k$, the bipartite graph $G\left[V_{i}, V_{j}\right]$ is $\varepsilon$-regular.

One feature of the Regularity Lemma, which unfortunately forbids practical applications, is that $k(\varepsilon)$ is very large: the best proof gives a tower of height about $1 / \varepsilon^{2}$, and unfortunately this is not far from the truth, as was shown by Gowers [60].

5.2. Weak Regularity Lemma and distance of graphs. A version with a weaker conclusion but with a more reasonable error bound was proved by Frieze and Kannan [52].

Lemma 5.2 (Weak Regularity Lemma). For every $k \geq 1$ and every graph $G=(V, E), V$ has a partition $\mathcal{P}$ into $k$ classes such that

$$
d_{\square}\left(G, G_{\mathcal{P}}\right) \leq \frac{2}{\sqrt{\log k}} .
$$


Note that we do not require here that $\mathcal{P}$ be an equipartition; it is not hard to see that this version implies that there is also an equipartition with similar property, just we have to increase the error bound to $4 / \sqrt{\log k}$.

To see the connection with the original lemma, we note that if $G$ is an $\varepsilon$-regular bipartite graph say in the sense of (36), and $H$ is the weighted complete bipartite graph with the same bipartition $\{U, W\}$ and with edge weights $d$, then (36) says that $d_{\square}(G, H) \leq \varepsilon$. Hence if $\mathcal{P}$ is a Szemerédi partition in the sense of Lemma 5.1, then the distance between the bipartite subgraph of $G$ induced by $V_{i}$ and $V_{j}$, and the corresponding weighted bipartite subgraph of $G_{\mathcal{P}}$, is at most $\varepsilon$ for all but $\varepsilon k^{2}$ pairs $(i, j)$, and at most 1 for the remaining $\varepsilon k^{2}$ pairs. It is easy to see that this implies that the distance between $G$ and $G_{\mathcal{P}}$ is at most $\varepsilon$. So the partition in Lemma 5.2 has indeed weaker properties than the partition in Lemma 5.1. Of course, this is compensated for by the relatively decent number of partition classes.

If we keep in $G_{\mathcal{P}}$ an edge with weight $p$ with probability $p$ and delete it with probability $1-p$, then we get a random graph $H$, and it is easy to see that with large probability $d_{\square}\left(G_{\mathcal{P}}, H\right) \leq \frac{10}{\sqrt{|V(G)|}}$. This implies the following version of the Weak Regularity Lemma:

Lemma 5.3. For every $k \geq 1$ and graph $G$, there is a graph $H$ with $k$ nodes such that

$$
\delta_{\square}(G, H) \leq \frac{10}{\sqrt{\log k}} .
$$

5.3. Strong Regularity Lemma and compactness. Other versions of the Regularity Lemma strengthen, rather than weaken, the conclusion (of course, at the cost of replacing the tower function by an even more formidable value). Such a "super-strong" Regularity Lemma was proved by Alon, Fisher, Krivelevich and Szegedy [5]. We state the following equivalent version from $[87]$.

Lemma 5.4 (Strong Regularity Lemma). For every sequence $\left(\varepsilon_{0}, \varepsilon_{1}, \ldots\right)$ of positive numbers there is a positive integer $k_{0}$ such that for every graph $G=(V, E)$, there is a graph $G^{\prime}$ on $V$, and $V$ has a partition $\mathcal{P}$ into $k \leq k_{0}$ classes such that

$$
d_{1}\left(G, G^{\prime}\right) \leq \varepsilon_{0} \quad \text { and } \quad d_{\square}\left(G^{\prime}, G_{\mathcal{P}}^{\prime}\right) \leq \varepsilon_{k} .
$$

Note that the first inequality involves the normalized edit distance, and so it is stronger than a similar condition with the cut distance would be. The second error bound $\varepsilon_{k}$ in (37) can be thought of as very small. If we choose $\varepsilon_{k}=\varepsilon_{0}$, we get the Frieze-Kannan version 5.2 (with $\varepsilon=2 \varepsilon_{0}$ ). Choosing $\varepsilon_{k}=\varepsilon_{0} / k^{2}$, the partition obtained satisfies the requirements of the original Regularity Lemma 5.1.

The strong version itself follows rather easily from the compactness of the space $\left(\mathcal{W}_{0}, \delta_{\square}\right)$ (Theorem 4.2$)$; see $[85]$ for details. 


\subsection{Partitions into sets with small diameter}

5.4.1. Small diameter sets and regularity. We can equip every graph $G=(V, E)$ with a metric as follows. Let $A$ be the adjacency matrix of $G$. We define the similarity distance $d_{2}(x, y)$ of two nodes $i, j \in V$ as the $\ell_{1}$ distance of the corresponding rows of $A^{2}$ (squaring the matrix seems unnatural, but it is crucial; it turns out to get rid of random fluctuations). The following was proved (in somewhat different form) in [87].

TheOREM 5.5. Let $G$ be a graph and let $\mathcal{P}=\left\{V_{1}, \ldots, V_{k}\right\}$ be a partition of $V$.

(a) If $d_{\square}\left(G, G_{\mathcal{P}}\right)=\varepsilon$, then there is a set $S \subseteq V$ with $|S| \leq 8 \sqrt{\varepsilon}|V|$ such that for each partition class, $V_{i} \backslash S$ has diameter at most $8 \sqrt{\varepsilon}$ in the $d_{2}$ metric.

(b) If there is a set $S \subseteq V$ with $|S| \leq \delta|V|$ such that for each partition class, $V_{i} \backslash S$ has diameter at most $\delta$ in the $d_{2}$ metric, then $d_{\square}\left(G, G_{\mathcal{P}}\right) \leq 24 \delta$.

Theorem 5.5 suggests to define the dimension of a family $\mathcal{G}$ of graphs as the infimum of real numbers $d>0$ for which the following holds: for every $\varepsilon>0$ and $G \in \mathcal{G}$ the node set of $G$ can be partitioned into a set of at most $\varepsilon|V(G)|$ nodes and into at most $\varepsilon^{-d}$ sets of diameter at most $\varepsilon$. (This number can be infinite.) In the cases when the graphs have a natural dimensionality, this dimension tends to give the right value. For example, let $G$ be obtained by selecting $n$ random points on the $d$-dimensional unit sphere, and connecting two of these points $x$ and $y$ with a probability $W(x, y)$, which is a continuous function of $x$ and $y$. With probability 1 , this sequence has dimension $\Theta(d)$.

5.4.2. Computational applications. As an easy application of Theorem 5.5, we give an algorithm to compute a weak Szemerédi partition in a huge graph. Our goal is to illustrate how an algorithm works in the pure sampling model, as well as in what form the result can be returned. This way of presenting the output of an algorithm for a large graph was proposed by Frieze and Kannan [52].

We start with an auxiliary algorithm that computes (approximately) the $d_{2}$ distance of two nodes.

Algorithm 5.6.

Input: A graph $G$ given by an sampling oracle, two nodes $u, v \in V$, and an error bound $\varepsilon>0$.

Output: A number $D_{2}(u, v) \geq 0$ such that with probability at least $1-\varepsilon$,

$$
D_{2}(u, v)-\varepsilon \leq d_{2}(u, v) \leq D_{2}(u, v)+\varepsilon .
$$

To see how this can be done, we rewrite the definition of the $d_{2}$ distance as follows. For $x, y \in V(G)$, let $a(x, y)$ be the corresponding entry of the 
adjacency matrix of $G$ : this is 1 if they are adjacent and 0 otherwise. Define

$$
a_{2}(x, y)=\mathrm{E}_{z} a(x, z) a(y, z),
$$

where $z$ is a uniform random node in $V$; this is the corresponding entry of the square of the adjacency matrix, normalized by $|V(G)|$. Finally, let

$$
d_{2}(x, y)=\mathrm{E}_{z}\left(\left|a_{2}(x, z)-a_{2}(y, z)\right|\right),
$$

where again $z$ is a uniform random node in $V$. Drawing a sufficiently large sample (depending on $\varepsilon$ ), these expectations can be approximated by averaging.

Algorithm 5.6 enables us to encode a partition of $V(G)$ as a subset $R \subseteq V(G)$ : for each $r \in R$, we define the partition class $V_{r}$ as the set of nodes $u \in V$ such that the node in $R$ closest to $u$ is $r$. Ties will be broken arbitrarily, and nodes to which there are several "almost closest" nodes may be misclassified, but this is the best one can hope for. To formalize,

Algorithm 5.7.

Input: A graph $G$ given by an sampling oracle, a subset $R \subseteq V(G)$, a node $u \in V$, and an error bound $\varepsilon>0$.

Output: An $r \in R$ such that with probability at least $1-\varepsilon, d_{2}(u, r) \leq$ $(1+\varepsilon) d_{2}(u, R)$.

The way this second algorithm works is that it uses Algorithm 5.6 to compute (approximately) the distances $d_{2}(u, r), r \in R$, and returns the node $r \in R$ that it finds closest to $u$. Borrowing a phrase from geometry, we compute the Voronoi cells of the set $R$.

Using this encoding of the partition, the following algorithm computes a weak Szemerédi partition.

Algorithm 5.8.

Input: A graph $G$ given by an sampling oracle, and an error bound $\varepsilon$.

Output: A set $R \subseteq V(G)$ with $|R| \leq 2^{2 / \varepsilon^{2}}$ such that, with probability at least $1-\varepsilon, d_{2}(u, R) \leq \varepsilon$ for all but an $\varepsilon$ fraction of the nodes $u$.

The set $R$ is grown step by step, starting with the empty set. At each step, a new uniform random node $w$ of $G$ is generated, and the approximate distances $D_{2}(u, v)$ are computed for all $r \in R$ with error less than $\varepsilon /|R|$. If all of these are larger than $\varepsilon / 2, w$ is added to $R$. Else, $w$ is thrown out and a new random node is generated. If $R$ is not increased in $1 / \varepsilon^{2}$ steps, the algorithm halts.

It is clear that if more than an $\varepsilon$ fraction of the nodes are farther than $\varepsilon$ from $R$, then in $1 / \varepsilon^{2}$ iterations we are very likely to sample one of these, and then with large probability we get the distances right and so we increase $R$.

Theorem 5.5 says in this context that the partition determined by Algorithms 5.6-5.8 satisfies $d_{\square}\left(G, G_{\mathcal{P}}\right) \leq(4 \varepsilon)^{1 / 4}$ with large probability. 
We conclude with an answer to Question 4 in Section 1.2. For the partition $\mathcal{P}$ implicitly determined above, we can also compute the edge densities between the partition classes, which we use to weight the edges of the complete graph on $R$, so that we get a weighted graph $H$. We find the maximum cut in $H$ by brute force, to get a partition $R=R_{1} \cup R_{2}$. This gives an implicit definition of a cut in $G$, where a node $u$ if put on the left side of the cut iff $D_{2}\left(u, R_{1}\right)<D_{2}\left(u, R_{2}\right)$ for the approximate distances computed by Algorithm 5.6.

5.5. Regularity Lemmas for bounded degree graphs? The Regularity Lemma as discussed above does not say anything for non-dense graphs. Several extensions for this case are known $[\mathbf{7 1}, \mathbf{5 4}]$, but they are meaningless for graphs that are very sparse, in particular if they have bounded degree.

Is there a Regularity Lemma for graphs with bounded degree? There are great difficulties here, but three results justify cautious optimism.

An observation of Alon (unpublished) implies that a weak analogue of the Regularity Lemma, version 5.3, holds. Using the sampling distance introduced in Section 1.3.1, we can state this as follows:

Proposition 5.9. For every $d \geq 1$ and $\varepsilon>0$ there is an $n=n(d, \varepsilon)$ such that for every graph $G$ with degrees bounded by $d$ there is a graph $H$ with degrees bounded by $d$ and $|V(H)| \leq n$, such that $d_{\text {sample }}(G, H) \leq \varepsilon$.

Unfortunately, no effective bound on $n$ follows from the proof. It would be very interesting to give any explicit bound on the function $n(d, \varepsilon)$, or to give an algorithm to construct $H$ from $G$. Ideally, one would like to design an algorithm that would work in the sampling framework, similarly as the algorithm in Section 5.4.2 works in the dense case.

It was proved recently by Elek and Lippner [41], and independently by Angel and Szegedy [11] that every graph with degrees bounded by $d$ can be decomposed by deleting $\varepsilon n$ edges into "highly homogeneous" parts, where the number of these parts is bounded by a function of $d$ and $\varepsilon$. Unfortunately, the highly homogeneous parts can still have a complex structure, but this may be a first important step in the direction of finding an analogue of the Regularity Lemma.

A third idea of decomposition is related to Følner sequences in the theory of amenable groups, and is called hyperfiniteness for general graph sequences $[40,102]$. A family $\mathcal{G}$ of graphs with bounded degree is called hyperfinite, if for every $\varepsilon>0$ there is a $k_{\varepsilon} \geq 1$ such that from every graph $G \in \mathcal{G}$ we can delete $\varepsilon|V(G)|$ edges so that every connected component of the remaining graph has at most $k_{\varepsilon}$ nodes. Schramm [102] showed that for a convergent graph sequence, hyperfiniteness is reflected by the limit object.

A special case of a hyperfinite family is a family $\mathcal{G}$ of graphs with subexponential growth, familiar from group theory. This property is defined by requiring that there is a function $f: \mathbb{N} \rightarrow \mathbb{N}$ such that $(\ln f(m)) / m \rightarrow 0$ $(m \rightarrow \infty)$, and for any graph $G \in \mathcal{G}$, any $v \in V(G)$ and any $m \in \mathbb{N}$, the number of nodes in the $m$-neighborhood of $v$ is at most $f(m)$. 
It is likely that large real-life networks can be thought of as hyperfinite; on the other hand, hyperfinite families of graphs seem to be much better behaved, and some of the theory of dense graph sequences can be extended at least to this case.

\section{Convergence and limits I: the dense case}

6.1. Subgraph sampling. Recall that we can define a notion of convergence if we fix a sampling method. For dense graphs, we use subgraph sampling: We select uniformly a random $k$-element subset of $V(G)$, and return the subgraph $G[k]$ induced by them. The probability that we see a given graph $F$ is the quantity $t_{\text {ind }}(F, G)$ introduced in (3). A sequence of graphs $\left(G_{n}\right)$ with $\left|V\left(G_{n}\right)\right| \rightarrow \infty$ is convergent if the induced subgraph densities $t_{\text {ind }}\left(F, G_{n}\right)$ converge for all finite graphs $F$.

We use this sampling method for dense graphs (otherwise all these densities tend to 0 ).

Instead of the induced subgraph densities $t_{\text {ind }}\left(F, G_{n}\right)$, we could use the subgraph densities $t_{\text {inj }}\left(F, G_{n}\right)$ or the homomorphism densities $t\left(F, G_{n}\right)$. Indeed, the subgraph densities can be expressed as linear combinations of induced subgraph densities and vice versa, while the difference $t(F, G)$ $t_{\text {inj }}(F, G)=O(1 /|V(G)|)$, and so it tends to 0 if $|V(G)| \rightarrow \infty$.

We can extend this sampling procedure to graphons, and we get to the construction of $W$-random graphs.

6.2. Convergence in distance. The definition of convergence can be reformulated using the notion of sampling distance 1: a sequence $\left(G_{n}\right)$ of simple graphs with $\left|V\left(G_{n}\right)\right| \rightarrow \infty$ is convergent if for every graph $F,\left(t_{\text {ind }}\left(F, G_{n}\right): i=1,2, \ldots\right)$ is a Cauchy sequence (equivalently, $\left(t\left(F, G_{n}\right): i=1,2, \ldots\right)$ is a Cauchy sequence). This is equivalent to saying that the graph sequence is Cauchy in the $d_{\text {sample }}$ metric. The following theorem, which is one of the main results in this theory, justifies the use of the cut metric $\delta_{\square}$.

THEOREM 6.1. A sequence $\left(G_{n}\right)$ of simple graphs $\left(\left|V\left(G_{n}\right)\right| \rightarrow \infty\right)$ is convergent if and only if it is a Cauchy sequence in the metric $\delta_{\square}$.

A quantitative form of this equivalence is given by the following theorem. Part (a) is a generalization of what is called the "Counting Lemma" in the theory of Szemerédi partitions; part (b) may be called the "Anti-counting" lemma.

Theorem 6.2. Let $U, W \in \mathcal{W}_{0}$.

(a) For every simple finite graph $F$,

$$
|t(F, U)-t(F, W)| \leq|E(F)| \cdot \delta_{\square}(U, W) .
$$

(b) Let $k$ be a positive integer, and assume that for every simple graph $F$ on $k$ nodes, we have

$$
|t(F, U)-t(F, W)| \leq 2^{-k^{2}} .
$$


Then

$$
\delta_{\square}(U, W) \leq \frac{20}{\sqrt{\log k}} .
$$

The proof of part (a) is quite simple; part (b) depends on the sampling lemmas to be discussed in Section 6.4.

Theorem 6.1 can be generalized to characterize convergence in the space $\mathcal{W}$ :

TheOrem 6.3. Let $\left(W_{n}\right)$ be a sequence of graphons in $\mathcal{W}_{0}$ and let $W \in$ $\mathcal{W}_{0}$. Then $t\left(F, W_{n}\right)$ converges for all finite simple graphs $F$ if and only if $W_{n}$ is a Cauchy sequence in the $\delta_{\square}$ metric. Furthermore $t\left(F, W_{n}\right) \rightarrow t(F, W)$ for all finite simple graphs $F$ if and only if $\delta_{\square}\left(W_{n}, W\right) \rightarrow 0$.

6.3. Convergence from the right. Convergence of a graph sequence can also be characterized in terms of mappings "to the right". Several characterizations along these lines were given in [30]; here we state one:

THEOREM 6.4. Let $\left(G_{n}\right)$ be a sequence of simple graphs such that $\left|V\left(G_{n}\right)\right| \rightarrow \infty$ as $n \rightarrow \infty$. Then the sequence $\left(G_{n}\right)$ is left-convergent if and only if the sequence $\mathcal{E}\left(G_{n}, H\right)$ is convergent for every weighted graph $H$.

6.4. Sampling and distance. The proof of the results in the previous section depends on a couple of probabilistic lemmas, which relate sampling to cut distance. The first of these lemmas is due to Alon, Fernandez de la Vega, Kannan and Karpinski [6], with an improvement from [29]. Its proof is quite involved. Its main implication is that the $d_{\square}$-distance of two graphs $G$ and $H$ on the same set of nodes can be estimated by sampling. It should be noted that the bound given is quite sharp.

Lemma 6.5. Let $k$ be a positive integer and let $G$ and $H$ be graphs with $V(G)=V(H),|V(G)| \geq k$ and edge weights in $[0,1]$. Let $S$ be chosen uniformly from all subsets of $V(G)$ of size $k$. Then with probability at least $1-2 e^{-\sqrt{k} / 8}$.

$$
\left|d_{\square}(G[S], H[S])-d_{\square}(G, H)\right| \leq \frac{10}{k^{1 / 4}} .
$$

The second lemma about sampling $[\mathbf{2 9}]$ shows that a sample is close to the original graph with large probability. Note that here we have to use the $\delta_{\square}$ distance (since no overlaying is given a priori), and also that the bound on the distance is much weaker than in the previous lemma.

Lemma 6.6. Let $k \geq 1$, and let $G$ be a simple graph on at least $k$ nodes. If $S$ is a random subset of $V(G)$ of size $k$, then with probability at least $1-2^{-k}$,

$$
\delta_{\square}(G, G[S]) \leq \frac{10}{\sqrt{\log k}} .
$$

This lemma follows from Lemma 6.5 and the Weak Regularity Lemma 5.2. Let us sketch this proof. 
Proof. Fix some $m \geq 1$. By Lemma 5.2 , there is an equipartition $\mathcal{P}=\left\{V_{1}, \ldots, V_{m}\right\}$ of $V(G)$ into $m$ classes such that

$$
d_{\square}\left(G, G_{\mathcal{P}}\right) \leq \frac{4}{\sqrt{\log m}} .
$$

Now let $S$ be a random $k$-subset. By Lemma 6.5 , we have

$$
\left|d_{\square}\left(G[S], G_{\mathcal{P}}[S]\right)-d_{\square}\left(G, G_{\mathcal{P}}\right)\right| \leq \frac{10}{k^{1 / 4}}
$$

with large probability. If $k$ is sufficiently large relative to $m$, then every class $V_{i}$ will contain about $k / m$ nodes from $S$.

Now blow up each node of $G_{\mathcal{P}}[S]$ into $n / k$ twins to get a weighted graph $G^{\prime}$ (in notation: $\left.G^{\prime}=G_{\mathcal{P}}[S]\left(\frac{n}{k}\right)\right)$. Then each set $V_{i} \cap S$ is blown up into a set $V_{i}^{\prime}$ of size $\frac{k}{n}\left|V_{i} \cap S\right| \approx\left|V_{i}\right|=\frac{n}{m}$.

It follows that we can overlay $G^{\prime}$ and $G_{\mathcal{P}}$ so that corresponding edges have the same weight except for edges inside the classes $V_{i}$ and edges incident with at most $\sum_{i=1}^{m}|| V_{i}^{\prime}|-| V_{i}||$ nodes. This is only a fraction of $\frac{1}{m}+\frac{4 m}{\sqrt{k}}$ of all edges, which shows that

$$
\delta_{\square}\left(G_{\mathcal{P}}, G_{\mathcal{P}}[S]\right)=\delta_{\square}\left(G_{\mathcal{P}}, G^{\prime}\right) \leq \frac{1}{m}+\frac{4 m}{\sqrt{k}} .
$$

Hence

$$
\begin{aligned}
\delta_{\square}(G, G[S]) & \leq \delta_{\square}\left(G, G_{\mathcal{P}}\right)+\delta_{\square}\left(G_{\mathcal{P}}, G_{\mathcal{P}}[S]\right)+\delta_{\square}\left(G_{\mathcal{P}}[S], G[S]\right) \\
& \leq \frac{4}{\sqrt{\log m}}+\frac{10}{k^{1 / 4}}+\left(\frac{1}{m}+\frac{4 m}{\sqrt{k}}\right) .
\end{aligned}
$$

Choosing $m=k^{1 / 4}$, we get

$$
\delta_{\square}(G, G[S]) \leq \frac{8}{\sqrt{\log k}}+\frac{10}{k^{1 / 4}}+\left(\frac{1}{k^{1 / 4}}+\frac{4}{k^{1 / 4}}\right)<\frac{10}{\sqrt{\log k}}
$$

if $k$ is large enough.

Both Lemmas 6.5 and 6.6 extend to graphons. We only formulate the second one, which can be stated in terms of the $W$-random graphs $\mathbb{G}(k, W)$.

Lemma 6.7. Let $k \geq 1$, and let $W$ be a graphon. Then with probability at least $1-2^{-k}$,

$$
\delta_{\square}(\mathbb{G}(k, W), W) \leq \frac{11}{\sqrt{\log k}} .
$$

To illustrate how these lemmas fit in the proofs, let us first sketch how Lemma 6.7 implies the "anti-counting lemma" (Theorem 6.2(b)). Assume that $U, W \in \mathcal{W}_{0}$ satisfy

$$
|t(F, U)-t(F, W)| \leq 2^{-k^{2}}
$$

for every graph $F$ with $k$ nodes. In terms of the $W$-random graphs $\mathbb{G}(k, U)$ and $\mathbb{G}(k, W)$, this implies (by inclusion-exclusion) that

$$
|\operatorname{Pr}(\mathbb{G}(k, U) \cong F)-\operatorname{Pr}(\mathbb{G}(k, W) \cong F)| \leq 2^{\left(\begin{array}{c}
k \\
2
\end{array}\right)} 2^{-k^{2}},
$$


and hence

$$
\sum_{F}|\operatorname{Pr}(\mathbb{G}(k, U) \cong F)-\operatorname{Pr}(\mathbb{G}(k, W) \cong F)| \leq 2^{k(k-1)} 2^{-k^{2}}=2^{-k} .
$$

This means that we can couple $\mathbb{G}(k, U)$ and $\mathbb{G}(k, W)$ so that $G(k, U) \cong$ $G(k, W)$ with probability at least $1-2^{-k}$. Lemma 6.7 implies that with probability at least $1-2^{-k}$, we have

$$
\delta_{\square}(U, \mathbb{G}(k, U)) \leq \frac{10}{\sqrt{\log k}},
$$

and similar assertion holds for $W$. Whenever all three happen, we get

$$
\begin{aligned}
& \delta_{\square}(U, W) \leq \delta_{\square}(U, \mathbb{G}(k, U))+\delta_{\square}(\mathbb{G}(k, U), \mathbb{G}(k, W))+\delta_{\square}(W, \mathbb{G}(k, W)) \\
& \quad \leq \frac{20}{\sqrt{\log k}} .
\end{aligned}
$$

6.5. Dense limit. The main motivation behind considering graphons is the following theorem $[\mathbf{8 5}]$ :

THEOREM 6.8. For any convergent sequence $\left(G_{n}\right)$ of simple graphs there exists a graphon $W$ such that $t\left(F, G_{n}\right) \rightarrow t(F, W)$ for every simple graph $F$.

We say that this graphon $W$ is the limit of the graph sequence, and write $G_{n} \rightarrow W$.

One might wonder if we really need complicated objects like integrable functions to describe these limits; would perhaps piecewise linear, or monotone, or continuous functions suffice? The following two results tell us that (up to weak isomorphism) all measurable functions are needed: every graphon $W$ can be obtained as the limit of a sequence of simple graphs [85], and the limit is essentially unique $[\mathbf{2 6}]$.

TheOREM 6.9. For any $W \in \mathcal{W}_{0}$, the graph sequence $\mathbb{G}(n, W)$ converges to the graphon $W$ with probability 1.

On the other hand, Theorem 3.1 implies:

THEOREM 6.10 ([26]). The limit graphon of a convergent graph sequence is uniquely determined up to weak isomorphism.

There are two quite different proofs of the (main) theorem 6.8. The original one in [85] uses Szemerédi partitions and the Martingale Convergence Theorem; a more recent proof by Elek and Szegedy [42] first constructs a different limit object in the form of an uncountable graph by taking the ultraproduct, and them obtains the graphon as an appropriate projection of this (in terms of non-standard analysis, the graphon is a non-standard Szemerédi partition of this graph on a non-standard $[0,1]$ interval).

The first proof has the obvious advantage of being a constructive; but the second proof is very general, it extends to hypergraphs and many other structures, and leads to new understanding of the Regularity Lemma for hypergraphs $[\mathbf{6 0}, \mathbf{6 1}, \mathbf{1 0 0}]$ and its consequences $[\mathbf{1 1 5}]$. 
Convergence to the limit object can also be characterized by the distance function introduced above [29]:

ThEOREM 6.11. For a sequence $\left(G_{n}\right)$ of graphs with $\left|V\left(G_{n}\right)\right| \rightarrow \infty$ and graphon $W$, we have $G_{n} \rightarrow W$ if and only if $\delta_{\square}\left(W_{G_{n}}, W\right) \rightarrow 0$.

Note that the function $W_{G_{n}}$ depends on the labeling of the nodes of $G_{n}$ (the distance $\delta_{\square}\left(W_{G_{n}}, W\right)$ does not, since relabeling $G_{n}$ results in weak isomorphism of $W_{G_{n}}$ ). Choosing the labeling appropriately, we can say more:

THEOREM 6.12. For a sequence $\left(G_{n}\right)$ of graphs with $\left|V\left(G_{n}\right)\right| \rightarrow \infty$ and graphon $W$, we have $G_{n} \rightarrow W$ if and only if the graphs $G_{n}$ can be labeled so that $\left\|W_{G_{n}}-W\right\|_{\square} \rightarrow 0$.

6.5.1. Equivalent descriptions of the limit. A random graph model is a probability distribution on simple graphs on $[n]$, for every $n \geq 1$, which is invariant under the reordering of the nodes. In other words, it is a sequence of random variables $\mathbb{G}_{n}$, whose values are simple graphs on $[n]$, and isomorphic graphs have the same probability. We say that a random graph model is consistent if deleting node $n$ from $\mathbb{G}_{n}$, the distribution of the resulting graph is the same as the distribution of $\mathbb{G}_{n-1}$. We say that the model is local, if for every $1<k<n$, the subgraphs of $\mathbb{G}_{n}$ induced by $[k]$ and $\{k+1, \ldots, n\}$ are independent as random variables.

It is easy to see that for every graphon $W \in \mathcal{W}_{0}, \mathbb{G}(n, W)$ is a consistent and local random graph model.

A related notion is the following. Let $\mathcal{G}$ be the set of graphs on $\mathbb{N}$; we can think of $\mathcal{G}$ as the product space $\{0,1\}^{E}$, where $E=\left(\begin{array}{c}\mathbb{N} \\ 2\end{array}\right)$ is the set of all (unordered) pairs of elements of $\mathbb{N}$. This also equips $\mathcal{G}$ with a $\sigma$-algebra. Let $\Sigma$ be the group of permutations of $\mathbb{N}$, and let $\Sigma_{2}$ be the action of $\Sigma$ on $E$. Recall that a probability measure $\pi$ on $\mathcal{G}$ is called ergodic with respect to $\Sigma_{2}$ if it is invariant under $\Sigma_{2}$ and $\mathcal{G}$ has no measurable subset $\mathcal{G}^{\prime}$ with $0<\pi\left(\mathcal{G}^{\prime}\right)<1$ invariant under $\Sigma_{2}$.

It is easy to see for that every $W \in \mathcal{W}$, the random graph $\mathbb{G}(W)$ defines a probability measure on $\mathcal{G}$ invariant under $\Sigma_{2}$. B. Szegedy $[\mathbf{1 1 1}, \mathbf{9 1}]$ showed that this measure is also ergodic.

After this preparation, we can formulate the theorem describing the many notions equivalent to graphons.

THEOREM 6.13. The following structures are cryptomorphic:

(a) a graphon $W \in \mathcal{W}_{0}$, up to weak isomorphism;

(b) A graph parameter $f$ that is the limit of graph parameters $t\left(., G_{n}\right)$ for some convergent graph sequence $\left(G_{n}\right)$.

(c) A multiplicative, reflection positive graph parameter $f$ satisfying $f\left(K_{1}\right)=1$,

(d) a consistent local random graph model;

(e) an ergodic measure on $\mathcal{G}$ invariant under $\Sigma_{2}$. 
The equivalences of these structures are mostly contained in results mentioned previously. Let us sketch these constructions.

$(\mathrm{a}) \rightarrow(\mathrm{b})$ : Every graphon $W \in \mathcal{W}_{0}$ gives rise to the graph parameter $t(., W)$; furthermore, $W$ is the limit of a convergent graph sequence $\left(G_{n}\right)$ (for example, of the sequence of $W$-random graphs), and for this sequence $t\left(F, G_{n}\right) \rightarrow t(F, W)$ for all $F$.

(b) $\rightarrow$ (c): If a graph parameter is the limit of graph parameters $t\left(., G_{n}\right)$, which satisfy the conditions in (c), then clearly so does their limit.

$(\mathrm{c}) \rightarrow(\mathrm{d})$ : In the special case when $f=t(., G)$ is the probability that a random map from $F$ to some graph $G$ is a homomorphism, we can express the probability that a sample of $n$ points gives a given graph $F_{0}$, by inclusionexclusion in terms of the numbers $f(F)$. We can apply the same formula to any graph parameter $f$ satisfying (c), and get a probability distribution on $n$-point graphs (here the conditions in (c) are used), which is a consistent local random graph model.

$(d) \rightarrow(a)$ : Generating a random graph $\mathbb{G}_{n}$ from the consistent local random graph model, it can be shown that we get a convergent graph sequence with probability 1 , which tends to a graphon $W$. For this graphon, $\mathbb{G}(n, W)$ gives back the random graph model we started with.

$(\mathrm{d}) \leftrightarrow(\mathrm{e})$ : It is easy to see that a consistent random graph model is equivalent to a probability distribution on $\mathcal{G}$ invariant under $\Sigma_{2}$. The proof that locality is equivalent to ergodicity $[\mathbf{1 1 1}, \mathbf{9 1}]$ is trickier and not given here.

COROLlaRY 6.14. A graph parameter $f$ is reflection positive if and only if it is either identically 0 , or there is a probability distribution $\rho$ on the Borel sets of $\left(\mathcal{W}_{0}, \delta_{\square}\right)$ such that if $\mathbf{W}$ denotes a random function from this distribution, then

$$
f(F)=\mathrm{E} t(F, \mathbf{W}) .
$$

6.5.2. Examples. We start with two easy examples.

EXAMPle 6.15. Complete bipartite graphs. It is natural to guess, and easy to prove, that complete bipartite graphs $K_{n, n}$ converge to the function defined by $W(x, y)=1$ if $0 \leq x \leq 1 / 2 \leq y \leq 1$ or $0 \leq y \leq 1 / 2 \leq x \leq 1$, and $W(x, y)=0$ otherwise.

ExAmple 6.16. Threshold graphs. These graphs are defined on the set $\{1, \ldots, n\}$ by connecting $i$ and $j$ if and only if $i+j \leq n$. These graphs converge to the function defined by $W(x, y)=\mathbf{1}_{x+y \leq 1}$.

EXAmPle 6.17. A sequence of graphs tending to the identically- $p$ function is exactly what we called a quasirandom sequence with density $p$.

Two examples of randomly growing graph sequences:

EXAMPLE 6.18. Randomly grown uniform attachment graph. We start with a single node. At the $n$-th iteration, a new node is born, and then 
every pair of nonadjacent nodes is connected with probability $1 / n$. We call this graph sequence a randomly grown uniform attachment graph sequence.

Let us do some simple calculations. After $n$ steps, let $\{0,1, \ldots, n-1\}$ be the nodes (born in this order). The probability that nodes $i<j$ are not connected is $\frac{j}{j+1} \cdot \frac{j+1}{j+2} \cdots \frac{n-1}{n}=\frac{j}{n}$. These events are independent for all pairs $(i, j)$. From here, one can easily figure out that the expected number of edges is $\left(n^{2}-1\right) / 6$.

To describe the limit function, note that the probability that nodes $i$ and $j$ are not connected is $\max (i, j) / n$. If $i=x n$ and $j=y n$, then this is $\max (x, y)$. Using that these events are independent, we can prove that the graph sequence $G_{n}^{\mathrm{ua}}$ tends to the limit function $1-\max (x, y)$ with probability 1.

EXAMPLE 6.19. Randomly grown prefix attachment graph. In this construction, it will be more convenient to label the nodes starting with 1 . At the $n$-th iteration, a new node $n$ is born, a node $z \leq n$ is selected at random, and the new node is connected to nodes $1, \ldots, z-1$. We denote the $n$-th graph in the sequence by $G_{n}^{\mathrm{pfx}}$, and call this graph sequence a randomly grown prefix attachment graph sequence.

The expected number of edges is $n(n-1) / 4$, and one can compute subgraph densities with some effort to see that the sequence is convergent with probability 1 . It is more difficult to figure out the limit graphon.

We can try to proceed similarly as in the case of uniform attachment graphs. The probability that $i$ and $j$ are connected is $|j-i| / \max (i, j)$; if $i=x n$ and $j=y n$, then this is $|x-y| / \max (x, y)$. Does this mean that the function $U(x, y)=|x-y| / \max (x, y)$ is the limit? Surprisingly, the answer is negative, which we can see by computing triangle densities.

The key to describe the limit is the remark at the end of Section 1.5.3, namely that instead of the uniform distribution over the interval $[0,1]$, we can use other probability spaces. Let us label a node born in step $k$, connected to $\{1, \ldots, m\}$, by the pair $(k / n, m / k) \in[0,1] \times[0,1]$. Then we can observe that nodes with label $\left(x_{1}, y_{1}\right)$ and $\left(x_{2}, y_{2}\right)$ are connected if and only if either $x_{1}<x_{2} y_{2}$ or $x_{2}<x_{1} y_{1}$.

From this observation one can prove that the prefix attachment graphs $G_{n}^{\mathrm{pfx}}$ converge, with probability 1 , to the function $W:[0,1]^{2} \times[0,1]^{2} \rightarrow[0,1]$, given by

$$
W_{\mathrm{pfx}}\left(\left(x_{1}, y_{1}\right),\left(x_{2}, y_{2}\right)\right)= \begin{cases}1, & \text { if } x_{1}<x_{2} y_{2} \text { or } x_{2}<x_{1} y_{1} \\ 0, & \text { otherwise }\end{cases}
$$

This gives a nice and simple representation of the limit object with the underlying probability space $[0,1]^{2}$ (with the uniform measure). If we want a representation on $[0,1]$, we can map $[0,1]$ into $[0,1]^{2}$ by any measure preserving map $\varphi$; then $W_{\mathrm{pfx}}^{\varphi}(x, y)=W^{\mathrm{pfx}}(\varphi(x), \varphi(y))$ gives a weakly isomorphic graphon. This function is $0-1$ valued, but its support is fractal-like. 
It is interesting to note that the graphs $\mathbb{G}(n, W)$ form a different growing sequence of random graphs tending to the same limit $W$ with probability 1 .

6.6. Convergence from the right. Paper [30] contains several conditions that characterize convergent dense graph sequences in terms of homomorphisms "to the right" (we have seen that these correspond to parameters with meaning in statistical physics). We only state one of these, in our terms:

THEOREM 6.20. Let $\left(G_{n}\right)$ be a sequence of graphs such that $\left|V\left(G_{n}\right)\right| \rightarrow$ $\infty$ as $n \rightarrow \infty$. Then the sequence $\left(G_{n}\right)$ is convergent if and only if the restricted multicut densities $\operatorname{rmcut}\left(G_{n}, H\right)$ are convergent for every weighted graph $H$.

By (11), the value rmcut in this theorem could be replaced by $\operatorname{hom}^{*}(G, H)$, and by our discussion in Section 2.3.3, we could talk about microcanonical ground state energies instead of restricted multicuts.

6.7. Limits of other dense combinatorial structures. Limit objects can be defined for multigraphs, directed graphs, colored graphs, hypergraphs etc. In many cases, like directed graphs without parallel edges, or graphs with nodes colored with a fixed number of colors, this can be done along the same lines as for simple graphs.

But in other cases there are some surprises. For example, limits of multigraphs with edge-multiplicities are not real valued functions, but 2-variable functions whose values are random variables with nonnegative integral values [89]. If $W$ is such a function, we can generate a $W$-random multigraph by selecting $n$ independent random points $X_{1}, \ldots, X_{n}$ from the uniform distribution on $[0,1]$, and then connecting nodes $i$ and $j$ with $W\left(X_{i}, X_{j}\right)$ parallel edges (which is a random integer).

The case of hypergraphs is much more interesting and important. Formulating regularity lemmas and constructing limits of sequences of $r$-uniform hypergraphs, where $r$ is fixed, is a highly nontrivial task, but it is essentially solved now, thanks to the work of Rödl and Skokan and Gowers $[\mathbf{1 0 0 , 5 9}]$; see also $[\mathbf{1 1 4}, \mathbf{4 2}]$.

However, it seems that no good extension of the distance $\delta_{\square}$ has been found to hypergraphs (just as for the regularity lemma, the first natural guesses are wrong). Another open question is to extend these results to nonuniform hypergraphs, with unbounded edge-size.

The semidefiniteness conditions for homomorphism functions can be extended to hypergraphs (see e.g. [80]). One area of applications of these conditions is extremal graph theory, and it is natural to ask if the semidefiniteness conditions can be useful in extremal hypergraph theory, especially since extremal problems for hypergraphs tend to be much harder than for graphs, and even basic questions are unsolved. 


\section{Convergence and limits II: bounded degree graphs}

7.1. Neighborhood sampling. Recall the sampling process for bounded degree graphs: For a fixed nonnegative integer $r$, we select uniformly a random node $v \in V(G)$, and return the ball $B_{G}(v, r)$ with center $v$ and radius $r$ (i.e., the subgraph induced by those nodes that can be reached from $v$ on a path of length $r$ or less). For a given rooted graph $F$, we denote by $\rho_{G, r}(F)$ the probability that this sampling method returns $F$ (with the root as the center). So $\rho_{G, r}(F)$ defines a probability distribution on rooted graphs $F$ with radius at most $k$, which we denote by $\rho_{G, r}$.

We use this method if the degrees of nodes in $G$ are bounded by a fixed number $d$; then the number of possible neighborhoods is finite.

A sequence of graphs $\left(G_{n}\right)$ with degrees uniformly bounded by $d$ and $\left|V\left(G_{n}\right)\right| \rightarrow \infty$ is convergent (or more precisely locally convergent) if the neighborhood densities $\rho_{G_{n}, r}(F)$ converge for all $r$ and all finite rooted graphs $F$.

Similarly as for the subgraph sampling, there are equivalent density type parameters whose convergence could be used instead of the neighborhood densities, for example, we could stipulate the convergence of $s\left(F, G_{n}\right)$ for every connected graph $F$.

\subsection{Local (weak) limit}

7.2.1. Different forms. A weakly convergent bounded degree graph sequence has several, not quite equivalent limit objects, which we have introduced in Section 3.2. Part (a) of the following theorem is due to Benjamini and Schramm [16]; part (b) was suggested by R. Kleinberg (unpublished); part (c), which implies (b), is due to Elek [37].

THEOREM 7.1. Let $\left(G_{n}\right)$ be a locally convergent sequence of graphs with degrees bounded by $d$. Then

(a) There is a unique unimodular distribution $\tau$ on countable rooted graphs with degrees bounded by d such that $\rho_{G_{n}, r} \rightarrow \rho_{\tau}$.

(b) There is a measure preserving graph $G$ such that $\rho_{G_{n}, r} \rightarrow \rho_{G, r}$ for every $k \geq 1$.

(c) There is a graphing $G$ such that $\rho_{G_{n}, r} \rightarrow \rho_{G, r}$ for every $k \geq 1$.

Note that in (b) we don't claim uniqueness. We could replace "graphing" by "measure preserving graph".

A big difference from the dense case is that there does not seem to be any easy way to construct a sequence that converges to a given graphing in this sense.

Conjecture 7.2 (Aldous-Lyons). Every graphing is the limit of a convergent sequence of bounded-degree graphs. Equivalently, every unimodular distribution on rooted countable graphs with bounded degree is the limit of a bounded degree graph sequence. 
7.2.2. Is the limit informative enough? The problem of the Regularity Lemma is related to conjecture 7.2. Indeed, suppose that we have a constructive way of finding, for an arbitrarily large graph $G$ with bounded degree, a graph $H$ of size bounded by a function of $r$ and $\varepsilon$ that approximates the distribution of $r$-neighborhoods in $G$ with error $\varepsilon$. The same construction should also work with a graphing instead of $G$. Letting $r \rightarrow \infty$ and $\varepsilon \rightarrow 0$, this would give a sequence of finite bounded degree graphs converging to the given graphing.

Part of the problem is to recognize "globally" when $H$ is a good approximation of $G$. Is there a good notion of "distance" (analogous to $\delta_{\square}$ ) for graphs with bounded degree?

The limit graphon of a dense sequence of graphs contains very much information about the asymptotic properties of the sequence. This is not so for the dense case, unfortunately.

PROBLEM 7.3. Is there a notion of convergence for graphs with bounded degree that is stronger than Benjamini-Schramm? (For example, one should be able to read off from the limit that the graphs are expanders.)

Let us illustrate this by a couple of simple examples.

EXAMPLE 7.4. Let $\left(G_{n}\right)$ be a sequence of 3-regular bipartite expander graphs with their girth tending to infinity. Let $H_{i}$ consist of two disjoint copies of $G_{i}$. The Benjamini-Schramm limit of both sequences is a distribution concentrated on a single 3 -regular rooted tree. In the Elek description, we get a graphing $\left(\Omega, T_{1}, T_{2}, T_{3}\right)$, where $T_{1}, T_{2}$ and $T_{3}$ generate a free group which acts on $\Omega$ without fixed points.

This limit graphing is not uniquely determined. One feels that in the case of the limit of the sequence $\left(G_{n}\right)$, the action of the free group should be ergodic, while in the case of the $H_{n}, \Omega$ should split into two invariant subsets of measure $1 / 2$. So it appears that in the limit object, the underlying $\sigma$-algebra also carries combinatorial information. This is in stark contrast with the dense case $[\mathbf{2 6}]$.

ExAmple 7.5. Let $G_{n}$ denote the $n \times n$ grid. The Benjamini-Schramm limit object is a probability distribution concentrated on the infinite grid with a specified root (the "origin"). A limit graphing can be described as the uniform measure on the 2-dimensional torus, together with the rotations by an irrational number $\alpha$ in one coordinate and the other.

However, in many respects the "right" limit object of the sequence of grids is a solid square. In other words, instead of larger and larger pieces of the infinite grid, we consider finer and finer subdivisions of the unit square.

This last example suggests that we can consider our graphs "on a different scale", and study them as metric spaces with the usual graph distance as metric, normalized by the diameter. We can then consider the limit of these metric spaces in the sense of Gromov [62]. For example, the limit of 
a sequence of larger and larger square grids in this sense is a (full) square. This global structure is not revealed by the Benjamini-Schramm limit.

It is easy to construct examples where the interesting structure of the graphs appears on an intermediate scale. It would be very interesting to describe and possibly unify limit objects belonging to different scales. Perhaps we can we understand different limit objects using ultraproducts, similarly to the work of Elek and Szegedy in the dense case.

7.3. Convergence from the right. While the description of convergent sequences in the bounded degree case lacks some of the key results that hold in the dense case, most notably a good notion of distance, we can formulate a result (Borgs, Chayes, Kahn and Lovász [25]) which shows that convergence defined in terms of homomorphisms from the left and homomorphisms to the right are equivalent under some circumstances.

To state this, let us define for every simple graph $G$ and weighted graph $H$ the quantity

$$
u(G, H)=\frac{\log \operatorname{hom}(G, H)}{|V(G)|},
$$

To see the meaning of $u(G, H)$, consider the case when $H$ is simple. Then $\operatorname{hom}(G, H) \leq q^{|V(G)|}$, and so after taking the logarithm and dividing by $|V(G)|$, we get a number less than $q$. So $u\left(G, K_{q}\right)$ expresses the freedom (entropy) we have in choosing the image of a node $v \in V(G)$ in a homomorphism $G \rightarrow H$.

For a weighted graph $H$, we define and

$$
\beta_{\max }=\max _{i, j} \beta_{i j}, \quad D(H)=\sum_{i, j \in V(H)} \frac{\alpha_{i} \alpha_{j}}{\alpha_{H}^{2}}\left(1-\frac{\beta_{i j}}{\beta_{\max }}\right) .
$$

THEOREM 7.6. Let $\left(G_{n}\right)$ be a sequence of graphs with maximum degree at most d.

(a) If $\left(G_{n}\right)$ is convergent, then for every weighted graph $H$ be a weighted graph with $D(H) \leq \frac{1}{2 d}$, the sequence $u\left(G_{n}, H\right)$ is convergent.

(b) Assume that for every $q \geq 1$ there is an $\varepsilon_{q}>0$ such that for every weighted graph $H$ on $q$ nodes with $D(H) \leq \varepsilon_{q}$ the sequence $q\left(G_{n}, H\right)$ is convergent. Then the sequence $\left(G_{n}\right)$ is convergent.

In the special case $H=K_{q}$ is the complete graph on $q$ nodes (without loops), we have $D\left(K_{q}\right)=1 / q$, and $\operatorname{hom}\left(G, K_{q}\right)$ is the number of $q$-colorings of $G$. So it follows that if $\left(G_{n}\right)$ is convergent and $q \geq 2 d$, then the number of $q$-colorations grows as $c^{\left|V\left(G_{n}\right)\right|}$ for some $c$. It is easy to see that some condition on $q$ is needed: for example, if $G_{n}$ is the $n$-cycle and $q=2$, then $q\left(G_{n}, K_{2}\right)$ oscillates between $-\infty$ and $\approx 0$ as a function of $n$. 


\section{Testing}

What can we learn about a huge graph $G$ from sampling? There are two related, but slightly different ways of asking this question, property testing and parameter estimation.

8.1. Sample concentration. Before discussing these tasks, let us address the following concern: if we take a bounded size sample from a graph, we can see very different graphs. For a random graph, for example, we can see anything. The natural way to use the sample $G[S]$ is to compute some graph parameter $f(G[S])$. But this parameter can vary wildly with the choice of the sample, so what information do we get?

The following two theorems assert that every reasonably smooth parameter of a sample is highly concentrated. (Note: we don't say anything here about the value of the parameter on the whole graph.)

The first version applies to parameters where smoothness is defined locally. The proof depends on the theory of martingales (Azuma's Inequality).

THEOREM 8.1. Let $f$ be a graph parameter and assume that $\mid f(G)-$ $f\left(G^{\prime}\right) \mid \leq 1$ for any two graphs on the same node set which differ only in edges incident with a single node. Then for every graph $G$ and $1 \leq k \leq|V(G)|$ there is a value $f_{0}$ such that if $S \subseteq V(G)$ is a random $k$-subset, then for every $t>0$,

$$
\left|f(G[S])-f_{0}\right|<\sqrt{2 t k}
$$

with probability at least $1-e^{-t}$.

The second version applies to parameters which are smooth with respect to our global distance function. The proof follows from a modification of the proof of Theorem 6.6.

THEOREM 8.2. Let $f$ be a graph parameter and assume that $\mid f(G)-$ $f\left(G^{\prime}\right) \mid \leq d_{\square}\left(G, G^{\prime}\right)$ for any two graphs on the same node set. Then for every graph $G$ and $1 \leq k \leq|V(G)|$ there is a value $f_{0}$ such that if $S \subseteq V(G)$ is a random $k$-subset, then

$$
\left|f(G[S])-f_{0}\right|<\frac{20}{\sqrt{k}}
$$

with probability at least $1-2^{-k}$.

8.2. Parameter estimation. We want to determine some parameter of a very large graph $G$. Of course, we'll not be able to determine the 
exact value of this parameter; the best we can hope for is that if we take a sufficiently large sample, we can find the approximate value of the parameter with large probability.

To be precise, a graph parameter $f$ is testable, if for every $\varepsilon>0$ there is a positive integer $k$ such that if $G$ is a graph with at least $k$ nodes and we select a set $X$ of $k$ independent uniform random nodes of $G$, then from the subgraph $G[X]$ induced by them we can compute an estimate $g(G[X])$ of $f$ such that

$$
\mathrm{P}(|f(G)-g(G[X])|>\varepsilon)<\varepsilon
$$

It is an easy observation that we can always use $g(G[X])=f(G[X])$ (cf. $[57])$.

It is easy to see that testability is equivalent to saying that for every convergent graph sequence $\left(G_{n}\right)$, the sequence of numbers $\left(f\left(G_{n}\right)\right)$ is convergent. (So graph parameters of the form $t(F,$.$) are testable by the defi-$ nition of convergence.) This is, however, more-or-less just a reformulation of the definition. Paper [29] contains a number of more useful conditions characterizing testability of a graph parameter. We formulate one, which is perhaps easiest to verify:

THEOREM 8.3. A graph parameter $f$ is testable if and only if the following three conditions hold:

(i) For every $\varepsilon>0$ there is an $\varepsilon^{\prime}>0$ such that if $G$ and $G^{\prime}$ are two simple graphs on the same node set and $d_{\square}\left(G, G^{\prime}\right) \leq \varepsilon^{\prime}$ then $\left|f(G)-f\left(G^{\prime}\right)\right| \leq \varepsilon$.

(ii) For every simple graph $G, f(G(m))$ has a limit as $m \rightarrow \infty$. (Recall that $G(m)$ denotes the graph obtained from $G$ by blowing up each node into $m$ twins.)

(iii) If $G^{+}$is obtained from $G$ by adding a single isolated node, then $f\left(G^{+}\right)-f(G) \rightarrow 0$ if $|V(G)| \rightarrow \infty$.

Note that all three conditions are special cases of the statement that

(iv) if $\left|V\left(G_{n}\right)\right|,\left|V\left(G_{n}^{\prime}\right)\right| \rightarrow \infty$ and $\delta_{\square}\left(G_{n}, G_{n}^{\prime}\right) \rightarrow 0$, then $f\left(G_{n}\right)-$ $f\left(G_{n}^{\prime}\right) \rightarrow 0$.

This condition is also necessary, so it is equivalent to its own three special cases (i)-(iii) in the theorem.

EXAMPlE 8.4. As a basic example, consider the density of maximum cuts (recall Section 2.3.2). One of the first substantial results on property testing $[\mathbf{5 6}, \mathbf{1 2}]$ is that this parameter is testable. It is relatively easy to see (using high concentration results like Azuma's inequality) that if $S$ is a sufficiently large random subset of nodes of $G$, then maxcut $(G[S]) \geq$ maxcut $(G)-\varepsilon$ : a large cut in $G$, when restricted to $S$, gives a large cut in $G[S]$. It is harder, and in fact quite surprising, that if most subgraphs $G[S]$ have a large cut, then so does $G$. This follows from Theorem 8.3 above, since conditions (i)-(iii) are easily verified for $f=$ maxcut. 
EXAMPLE 8.5. The free energy (16) for a fixed weighted graph $H$ is a more complicated example of a testable parameter, which illustrates the power of Theorem 8.3. It is difficult to verify directly either the definition, or say condition (iv). The theorem splits this into three: condition (i) is easy by the definition of $d_{\square}\left(G, G^{\prime}\right)$; (ii) is a matter of classical combinatorics, counting mappings that split the twin classes in given proportions; finally, (iii) is trivial.

8.3. Dense property testing. Instead of estimating a numerical parameter, we may want to determine some property of $G$ : Is $G 3$-colorable? Is it connected? Does it have a triangle? The answer will of course have some uncertainty. A precise definition was given by Rubinfeld and Sudan [101] and Goldreich, Goldwasser and Ron [56]. In the slightly different context of "additive approximation", closely related problems were studied by Arora, Karger and Karpinski [12] (see e.g. [45] for a survey). Many extensions deal with situations where we are allowed to sample more than a constant number of nodes of the large graph $G$; our concern will be the original setup, where the sample size is bounded.

A graph property $\mathcal{P}$ is testable, if there exists another property $\mathcal{P}^{\prime}$ (called a "test property") such that

(a) if a graph $G$ has property $\mathcal{P}$, then for all $1 \leq k \leq|V(G)|$ at least $2 / 3$ of its $k$-node induced subgraphs have property $\mathcal{P}^{\prime}$, and

(b) for every $\varepsilon>0$ there is a $k_{\varepsilon} \geq 1$ such that if $G$ is a graph whose edit distance from $\mathcal{P}$ is at least $\varepsilon|V(G)|^{2}$, then for all $k_{\varepsilon} \leq k \leq|V(G)|$ at most a fraction of $1 / 3$ of the $k$-node induced subgraphs of $G$ have property $\mathcal{P}^{\prime}$.

This notion of testability is usually called oblivious testing, which refers to the fact that no information about the size of $G$ is assumed. The constants $1 / 3$ and $2 / 3$ are arbitrary, and it would not change the notion of testability if we replaced them by any two real numbers $0<a<b<1$.

It is surprising that this rather restrictive definition allows many testable graph properties: for example, bipartiteness, triangle-freeness, every property definable by a first order formula [5].

A surprisingly general result was proved by Alon and Shapira [8]. A graph property $\mathcal{P}$ is called hereditary if $G \in \mathcal{P}$ implies that $G^{\prime} \in \mathcal{P}$ for every induced subgraph $G^{\prime}$ of $G$.

TheOREM 8.6 (Alon-Shapira). Every hereditary graph property is testable.

Fischer and Newman [46] proved that a property is testable if and only if the normalized edit distance from the property is a testable parameter. Alon et al. characterized testable graph properties in terms of Szemerédi partitions [7]. 
Going to the limit gives a tool of studying testability in a "cleaner" form (Lovász and Szegedy [88]). It turns out that this leads to an interesting interplay between the cut-norm and the $L_{1}$-norm on $\mathcal{W}_{0}$.

A graph property $\mathcal{P}$ can be thought of as a subset of $\mathcal{W}_{0}$ (through the correspondence $G \mapsto W_{G}$ ), and we can consider its closure $\overline{\mathcal{P}}$ in the metric space $\left(\mathcal{W}_{0}, \delta_{\square}\right)$. For example, the closure of the set of triangle-free graphs is the set of triangle-free graphons, which can be characterized by the property $t\left(K_{3}, W\right)=0$. More generally, let $\mathcal{P}$ be a hereditary graph property. Then its closure is characterized by the (infinitely many) equations

$$
t_{\text {ind }}(F, W)=0 \quad \text { for all } \quad F \notin \mathcal{P} .
$$

Closures of testable graph properties will be called testable graphon properties. These graphon properties can also be characterized in terms of a sampling method: we consider the $W$-random graph $\mathbb{G}(k, W)$ as the sample of size $k$ from $W$.

THEOREM 8.7. A graphon property $\mathcal{R}$ is testable if and only if there is a graph property $\mathcal{R}^{\prime}$ such that

(a) $\operatorname{Pr}\left(\mathbb{G}(k, W) \in \mathcal{R}^{\prime}\right) \geq 2 / 3$ for every function $W \in \mathcal{R}$ and every $k \geq 1$, and

(b) for every $\varepsilon>0$ there is a $k_{\varepsilon} \geq 1$ such that $\operatorname{Pr}\left(\mathbb{G}(k, W) \in \mathcal{R}^{\prime}\right) \leq 1 / 3$ for every $k \geq k_{\varepsilon}$ and every function $W \in \mathcal{W}_{0}$ with $d_{1}(W, \mathcal{R}) \geq \varepsilon$.

We quote an analytic characterization of testable graphon properties [88]. Recall that the distances $d_{1}$ and $d_{\square}$ are related trivially by $d_{\square} \leq d_{1}$. Testability of a property concerns an inverse relation:

THEOREM 8.8. A graphon property $\mathcal{R}$ is testable if and only if either one of the following conditions hold:

(a) For every $\varepsilon>0$ there is an $\varepsilon^{\prime}>0$ such that if $d_{\square}(W, \mathcal{R}) \leq \varepsilon^{\prime}$ for some graphon $W$, then $d_{1}(W, \mathcal{R}) \leq \varepsilon$.

(b) $d_{1}(W, \mathcal{R})$ is a continuous function of $W$ in the cut norm.

Condition (b) can be viewed as the graphon analogue of the theorem of Fischer and Newman mentioned above (and the finite theorem can be derived from it). Condition (a) is a special case of (b).

ExAmple 8.9. Let $\mathcal{R}=\{U\}$, where $U \in \mathcal{W}$ is the identically $1 / 2$ function. Clearly this property is invariant under weak isomorphism. Consider the random graphs $G_{n}=\mathbb{G}(n, 1 / 2)$; then $\left\|W_{G_{n}}-U\right\|_{\square} \rightarrow 0$ with probability 1 , but $\left\|W_{G_{n}}-U\right\|_{1}=1 / 2$ for every $n$. So this property is not testable by Theorem 8.8.

Let us sketch how the graphon version of Theorem 8.6 follows from this. A property $\mathcal{R}$ of functions $W \in \mathcal{W}_{0}$ is called flexible if for every function $U$ such that $U(x, y)=W(x, y)$ for all $x, y$ with $W(x, y) \in\{0,1\}$, we also have $U \in \mathcal{R}$. First, one proves that 
Lemma 8.10. The closure of a hereditary property is flexible.

Indeed, each of the equations (38) is preserved if we change the value of $W$ at points where this value is positive.

Next, we assume that $\mathcal{R}$ is a closed flexible property which is not testable. By Theorem 8.8, there is a sequence of functions $W_{n}$ such that $d_{\square}\left(W_{n}, \mathcal{R}\right) \rightarrow$ 0 but $d_{1}\left(W_{n}, \mathcal{R}\right) \geq \varepsilon$ for some fixed $\varepsilon>0$. By Theorem 4.2 , we may assume that $W_{n}$ converges to some $W \in \mathcal{R}$ in the $\|.\|_{\square}$ norm. Let $S_{0}=W^{-1}(0)$, $S_{1}=W^{-1}(1)$ and let $Z_{n} \in \mathcal{W}_{0}$ denote the function which is 1 on $S_{1}$, 0 on $S_{0}$ and is identical with $W_{n}$ anywhere else. By flexibility, we have $Z_{n} \in \mathcal{R}$, and by (34),

$$
\left\|W_{n}-Z_{n}\right\|_{1}=\int_{S_{0}} W_{n}+\int_{S_{1}}\left(1-W_{n}\right) \rightarrow \int_{S_{0}} W+\int_{S_{1}}(1-W)=0 \quad(n \rightarrow \infty),
$$

and so $d_{1}\left(W_{n}, \mathcal{R}\right) \rightarrow 0$, a contradiction. So it follows that the closure of every hereditary property is testable.

From this, one can derive that hereditary properties are testable. Some further arguments are needed, since a graph property can have a testable closure without itself being testable. (An example is the property that the graph is complete if the number of nodes is even but edgeless if the number of nodes is odd.) One can add further conditions that lead to a characterization, but we don't go into these technical issues here.

8.4. Sparse property testing. We say that a graph property $\mathcal{P}$ is testable for graphs in $\mathcal{G}_{d}$ if for every $\varepsilon>0$ there are integers $r=r(d, \varepsilon) \geq 1$ and $k=k(d, \varepsilon)$ such that sampling $k$ neighborhoods of radius $r$ from a graph $G$ with degree bounded by $d$, we can compute "YES" or "NO" so that:

(a) if we answer "NO", then $G \notin \mathcal{P}$;

(b) if we answer "YES", then we can change at most $\varepsilon|V(G)|$ edges in $G$ to get a graph in $\mathcal{P}$.

An important analogue of the result of Alon and Shapira discussed above is the following theorem of Benjamini, Schramm and Shapira [17]. We must recall a fundamental notion from graph theory: a minor of a graph $G$ is any other graph obtained from $G$ by deleting edges and/or nodes, and contracting edges. A graph property is minor-closed, if it is preserved by these operations. Planarity of a graph is an example of a minor-closed property.

THEOREM 8.11. Every minor-closed property is testable for graphs with bounded degrees.

A related result was proved by Elek [38]:

THEOREM 8.12. If a graph property is preserved by edge/node deletion and disjoint union, then it is testable for graphs with bounded degrees and subexponential growth. 


\section{Extremal graph theory}

9.1. Some classical results. In this section we describe applications of the theory of graph homomorphisms and graph limits to extremal graph theory. As an introduction, let us recall some classical results.

Define the Turán graph $T(n, r)(1 \leq r \leq n)$ as follows: we partition $[n]$ into $r$ classes as equitably as possible, and connect two nodes if and only if they belong to different classes.

Theorem 9.1 (Turán's Theorem). Among all graphs on $n$ nodes containing no $K_{k}$, the graph $T(n, k-1)$ has the maximum number of edges.

Since we are interested in large $n$ and fixed $k$, the complication that the classes cannot be exactly equal in size (which causes the formula for the number of edges of $T(n, k-1)$ to be a bit ugly) should not worry us. We will be interested in the following corollary:

COROLlary 9.2. If a graph on $n$ nodes has more than $\left(\begin{array}{c}k-1 \\ 2\end{array}\right)\left(\frac{n}{k-1}\right)^{2}$ edges, then it contains a $K_{k}$.

The case $k=3$ was proved by Mantel before Turán. We will use this case to illustrate the ideas, but the general case could be treated similarly.

One can ask for not just the existence of complete $k$-graphs, but for their number. Generalizing Turán's Theorem, the following lower bound was proved by Goodman (for $k=3$ ) and by Moon and Moser.

THEOREM 9.3. If a graph on $n$ nodes has $a\left(\begin{array}{c}n \\ 2\end{array}\right)$ edges $(0 \leq a \leq 1)$, then it contains at least $a(2 a-1) \ldots((k-2) a-k+1)\left(\begin{array}{l}n \\ k\end{array}\right)$ complete $k$-graphs.

This bound is tight for Turán graphs, but their edge density attains only certain values of $a$. The best lower bound in terms of $a$ and $n$ is quite complicated. To illustrate these complications, we represent each graph $G$ by the points $\left(t\left(K_{2}, G\right), t\left(K_{3}, G\right)\right.$ in the unit square (see Figure 2). The lower bounding curve consists of infinitely many concave cubic arcs, and its validity was only recently proved by Razborov $[\mathbf{9 8}]$. This was extended to

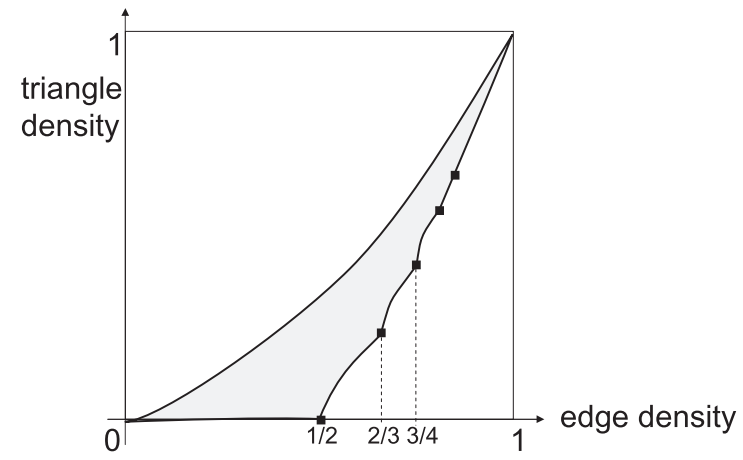

Figure 2. Possible edge and triangle densities of a graph 
the best lower bound on the number of $K_{4}$-s by Nikiforov [95], but even the edge $-K_{q}$ diagram is only conjectural $[\mathbf{8 3}]$ for $q \geq 5$.

One can also ask for an upper bound on the number of complete $k$-graphs in a graph with given number of edges. A special case of the Kruskal-Katona Theorem answers this (the whole theorem gives the precise value, not just asymptotics, and concerns uniform hypergraphs, not just graphs).

THEOREM 9.4. If a graph on $n$ nodes has $a\left(\begin{array}{l}n \\ 2\end{array}\right)$ edges $(0 \leq a \leq 1)$, then it contains at most $a^{k / 2}\left(\begin{array}{l}n \\ k\end{array}\right)$ complete $k$-graphs.

Asymptotic equality is attained when the graph consists of a clique and isolated nodes. Not every edge density $a$ can be realized by such graphs, but the attainable edge densities are dense in $[0,1]$, and so Theorem 9.4 is asymptotically tight for all values of $a$.

Instead of counting complete graphs, we one can consider the number of copies of some other graph $F$ in $G$. We have already come across counting 4-cycles twice: in Section 1.4.3 and in Section 1.5.4. Giving just the simpler asymptotic version:

TheOREM 9.5 (Erdös). If a graph on n nodes has a $\left(\begin{array}{l}n \\ 2\end{array}\right)$ edges $(0<a \leq 1)$, then it contains at least $\left(\frac{1}{8}+o(1)\right) a^{4} n^{4} 4$-cycles.

Graphs with asymptotic equality here are quasirandom graphs.

The number of paths of length $k$ is a more difficult question, but it turns out to be equivalent to a theorem of Blakley and Roy [18] in matrix theory. Again asymptotically,

THEOREM 9.6. If a graph on $n$ nodes has $a\left(\begin{array}{l}n \\ 2\end{array}\right)$ edges $(0<a \leq 1)$, then it contains at least $\left(\frac{1}{2}+o(1)\right) a^{k-1} n^{k}$ paths of length $k$.

Regular graphs give asymptotic equality here.

9.2. Algebraic proofs of extremal graph results. The classical extremal problems in the previous section can be expressed as algebraic inequalities between the subgraph densities $t(F, W)$ that hold for all graphons $W$. Often "going to the infinity" provides cleaner formulations (no error terms). Here are a few examples:

\section{EXAMPLE 9.7.}

(a) Turán's theorem. We state just the case of triangles (due to Mantel):

$$
t\left(K_{3}, W\right)=0 \Rightarrow t\left(K_{2}, W\right) \leq 1 / 2,
$$

which follows from the algebraic inequality due to Goodman [58]:

$$
t\left(K_{3}, W\right) \geq t\left(K_{2}, W\right)\left(2 t\left(K_{2}, W\right)-1\right) .
$$

(b) The Kruskal-Katona theorem for graphs:

$$
t\left(K_{3}, W\right) \leq t\left(K_{2}, W\right)^{3 / 2} .
$$


(c) Erdös's bound on the number of quadrilaterals:

$$
t\left(C_{4}, W\right) \geq t\left(K_{2}, W\right)^{4} .
$$

(d) The Blakley-Roy inequality:

$$
t\left(P_{k}, W\right) \geq t\left(K_{2}, W\right)^{k-1} .
$$

(e) The Sidorenko Conjecture (unsolved) generalizes the last two results in the direction that for every bipartite graph $F$,

$$
t(F, W) \geq t\left(K_{2}, W\right)^{|E(F)|} .
$$

This conjecture is proved for trees, many small graphs, complete bipartite graphs (Sidorenko [107]) and also for cubes (Hatami [64]).

Using the formalism introduced above, the results in Example 9.7 can be expressed as follows:

(a) $K_{3} \geq 2 K_{2}^{2}-K_{2}$;

(b) $K_{2}{ }^{3} \geq K_{3}{ }^{2}$;

(c) $C_{4} \geq K_{2}^{4}$;

(c) $P_{4} \geq K_{2}^{3}$;

(d) $F \geq K_{2}|E(F)| \quad$ (if $F$ is bipartite).

The first three inequalities can be proved easily using the reflection positivity of the graph parameters $t(., W)$. We will illustrate the method by deriving (a) through formal algebraic manipulations.

Proof of (a) (Goodman's extension of the Mantel-Turán Theorem). Let $F$ denote the graph $K_{2} K_{1}$ (an edge and an isolated node), and let $F_{1}, F_{2}$ and $F_{3}$ be obtained from $F$ by labeling all three nodes, one endpoint of the edge, and the isolated node, respectively. Consider the quantum graph ${\widetilde{F_{1}}}^{2}+2\left(F_{2}-F_{3}\right)^{2}$, which is obviously nonnegative. Unlabeling the nodes and deleting isolated nodes, we get $K_{3}-2 K_{2}^{2}+K_{2}$, which is thus nonnegative (see Figure 3).

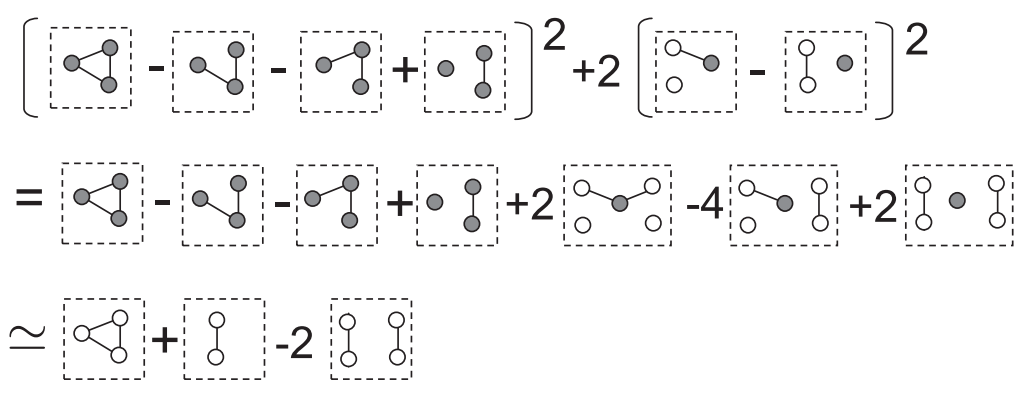

Figure 3. A computation proving the Mantel-Turán Theorem 
Of the above inequalities, also (b) and (c) can be proved by similar arguments. The Blakley-Roy inequality (c) is more difficult, but some extension of this kind of argument does work [74]. Sidorenko's conjecture (d) would of course be very nice to prove this way (or by any other means).

Using related methods, Razborov [98] solved the long-standing problem of characterizing the possible (edge-density, triangle-density) pairs, which in this setting means a description of the set $\left.\left(t\left(K_{2}, W\right), t\left(K_{3}, W\right)\right): W \in \mathcal{W}_{0}\right)$ by algebraic inequalities.

The inequality in (c) also follows from reflection positivity if $k$ is even. It is not known whether (c) for odd $k$ (or perhaps every valid algebraic inequality between subgraph densities) follows from a finite number of semidefiniteness inequalities. However, every valid linear inequality between homomorphism densities follows from semidefiniteness constraints (equivalently, from "sums of squares" computations in graph algebras), as we shall see in the next section.

9.3. Positivstellensatz for graphs and spectral norms. The machinery introduced in the previous sections allows us to suggest a very general approach to extremal graph theory.

We can define the following partial order on $\mathcal{Q}_{0}$ : we say that a quantum graph $x \geq 0$, if $t(x, W) \geq 0$ for all $W \in \mathcal{W}_{0}$.

Let us call a quantum graph $y$ a square-sum if there are $k$-labeled quantum graphs $y_{1}, \ldots, y_{k}$ for some $k$ such that $y$ can be obtained from $\sum_{i} y_{i}^{2}$ by forgetting the labels. It is easy to see that every square-sum satisfies $y \geq 0$.

As an example, recall the definition (18) of the "inclusion-exclusion" quantum graph $\widehat{F}$. Let us label all nodes of $\widehat{F}$, square it, and then forget the labels: we obtain $\widehat{F}$ itself. This implies that $\widehat{F} \geq 0$ for all $F$. In the special case when $W=W_{G}$ for some graph $G$, this also follows from our previous remark that $t(\widehat{F}, G)$ is a probability, and hence nonnegative.

Is there a quantum graph $x \geq 0$ which is not a square sum? I suspect that such quantum graphs exist, but it might be difficult to prove this property. However, the following weaker result can be proved $[\mathbf{9 1}]$.

THEOREM 9.8. Let $x$ be a quantum graph. Then $x \geq 0$ if and only if for every $\varepsilon>0$ there is a square-sum $y$ such that $N(y) \leq N(x)$ and $\|x-y\|_{2}<\varepsilon$.

The proof depends on the duality theory of semidefinite programs. Note that we do not claim that the $k$-labeled quantum graphs $y_{i}$ in the squaresum representation of $y$ also have bounded $N\left(y_{i}\right)$; the proof gives arbitrarily large graphs if $\varepsilon$ is small.

In analogy with the Positivstellensatz for real polynomials, we may try to represent quantum graphs $x \geq 0$ as quotients of square-sums: if $y$ and $z$ are square-sums and $y=z x$, then $x \geq 0$.

We mention a couple of related questions. For every even positive integer $k$, the functional $t\left(C_{k}, W\right)^{1 / k}$ defines a norm on $\mathcal{W}$ (the Neumann-Schatten norm). This suggests the question: For which other simple graphs $F$ is 
$t(F, W)^{1 /|E(F)|}$ a norm (or seminorm) on $\mathcal{W}$ ? Hatami $[\mathbf{6 4}]$ proved that if a simple graph $F$ has the property that $\|W\|=t(F,|W|)^{1 /|E(F)|}$ is a norm, then it satisfies Sidorenko's conjecture 9.7(d). He also proved that all cubes have this property.

In view of the usefulness of extending graphs to graphons, it seems natural to define graph algebras of infinite linear combinations of graphs with appropriate convergence properties. It is not worked out, however, what the structure of the resulting algebra is, and how it is related to graphons.

9.4. The maximum distance from a hereditary graph property. A surprisingly general result is the theorem of Alon and Stav $[\mathbf{9}]$, proving that for every hereditary property, a random graph with appropriate density is asymptotically the farthest from the property in edit distance. The analytic results developed in this paper allow us to state and prove a simple analytic analogue of this fact, from which the original result follows along with generalizations.

TheOREM 9.9 (Alon and Stav). For every hereditary graph property $\mathcal{P}$ there is a number $p, 0 \leq p \leq 1$, such that for every graph $G$ with $|V(G)|=n$,

$$
d_{1}(G, \mathcal{P}) \leq \mathrm{E}\left(d_{1}(\mathbb{G}(n, p), \mathcal{P})\right)+o(1) \quad(n \rightarrow \infty) .
$$

The following theorem [88] states a graphon version of this fact.

THEOREM 9.10. If $\mathcal{R}$ is the closure of a hereditary graph property, then the maximum of $d_{1}(., \mathcal{R})$ is attained by a constant function.

Our point in giving this generalization is to illustrate the power of extending graph problems to a continuum. The key observation is the following, which follows from Lemma 8.10.

LEMMA 9.11. If $\mathcal{R}$ is the closure of a hereditary graph property, then the set $\mathcal{W}_{0} \backslash \mathcal{R}$ is convex.

Hence it follows that the $d_{1}$ distance from $\mathcal{P}$ is a concave function on $\mathcal{W}_{0} \backslash \mathcal{R}$. Since $\mathcal{W}_{0} \backslash \mathcal{R}$ is obviously invariant under the group of invertible measure preserving transformations of $[0,1]$, it is not hard to argue that there is a point (graphon) in $\mathcal{W}_{0} \backslash \mathcal{R}$ maximizing the distance from $\mathcal{P}$ which is invariant under these measure preserving transformations, and so it must be a constant function.

9.5. Which graphs are extremal? (Finitely forcible graphons). We call a graphon $W \in \mathcal{W}_{0}$ finitely forcible if there exist a finite list of graphs $F_{1}, \ldots, F_{m}$ and real numbers $a_{1}, \ldots, a_{m}$ such that the equations $t\left(F_{1}, U\right)=$ $a_{1}, \ldots, t\left(F_{m}, U\right)=a_{m}$ are satisfied by precisely those functions $U \in \mathcal{W}_{0}$ which arise from $W$ by measure preserving transformations. 
Let us consider a very general type of graph theoretic extremal problem:

$$
\begin{aligned}
& \operatorname{maximize} t(f, W) \\
& \text { subject to } t\left(g_{1}, W\right)=a_{1} \\
& t\left(g_{2}, W\right)=a_{1} \\
& \vdots \\
& t\left(g_{k}, W\right)=a_{1}
\end{aligned}
$$

where $f, g_{1}, \ldots, g_{k}$ are given quantum graphs. Most of the graphon versions of extremal problems discussed so far fit in this scheme.

It is easy to see that every finitely forcible graphon is the solution of an extremal problem of the type (45). We conjecture the following converse:

Conjecture 9.12. Every extremal problem has a finitely forcible optimum. In other words, if a finite set of constraints of the form $t\left(F_{i}, W\right)=a_{i}$ is satisfied by some graphon, then it is satisfied by a finitely forcible graphon.

This may seem far fetched, but the following heuristic supports it. Suppose that $t\left(F_{1}, W\right)=a_{1}, \ldots, t\left(F_{k}, W\right)=a_{k}$ has a solution in $W$, but this is not forced by these constraints. Then there is a graph $F$ such that $t(F, W)$ is not determined, i.e., $a=\min t(F, W)<\max t(F, W)=b$ (the $\max$ and min are taken over all solutions $W$ of the system). Now add one of the conditions $t(F, W)=a$ or $t(F, W)=b$ to the system and repeat. It seems that in very few $(2-3)$ steps we always get a unique solution, i.e., a finitely forcible graphon.

Almost all classical extremal problems have a solution that is a stepfunction. It was shown by Lovász and Sós [84] that every stepfunction is finitely forcible, and it was conjectured that these are the only ones. Recently B. Szegedy and Lovász [90] found other finitely forcible graphons, and so the problem of characterizing finitely forcible graphons is wide open.

We mention two examples of finitely forcible graphons that are not stepfunctions (the proof is not quite easy).

EXAMPLE 9.13. Let $p(x, y)$ is a symmetric real polynomial that is monotone increasing on $[0,1]^{2}$. Define

$$
W(x, y)= \begin{cases}1, & \text { if } p(x, y)>0 \\ 0, & \text { otherwise }\end{cases}
$$

Then $W$ is finitely forcible. It is conjectured that monotonicity is not needed here.

In contrast, one can show that if $W(x, y)$ is a polynomial in $x$ and $y$ (not a function of the sign), then it is not finitely forcible. 
EXAMPLE 9.14. Let

$$
W(x, y)= \begin{cases}1, & \text { if the first bit where the binary expansions of } x \text { and } y \\ & \text { differ is at an odd position, } \\ 0, & \text { otherwise }\end{cases}
$$

The $W$ is finitely forcible.

\section{References}

[1] R. Albert, A.-L. Barabási: Statistical mechanics of complex networks, Rev. Modern Phys. 74 (2002), 47-97.

[2] D.J. Aldous: Tree-valued Markov chains and Poisson-Galton-Watson distributions, in: Microsurveys in Discrete Probability (D. Aldous and J. Propp, editors), DIMACS Ser. Discrete Math. Theoret. Comput. Sci. 41 (1998) Amer. Math. Soc., Providence, RI. (1998), 1-20.

[3] D. Aldous, R. Lyons: Processes on Unimodular Random Networks, Electron. J. Probab. 12, Paper 54 (2007), 1454-1508.

[4] D.J. Aldous and M. Steele: The Objective Method: Probabilistic Combinatorial Optimization and Local Weak Convergence, in: Discrete and Combinatorial Probability (H. Kesten, ed.), Springer (2003) 1-72.

[5] N. Alon, E. Fischer, M. Krivelevich and M. Szegedy: Efficient testing of large graphs, Combinatorica 20 (2000) 451-476.

[6] N. Alon, W. Fernandez de la Vega, R. Kannan and M. Karpinski: Random sampling and approximation of MAX-CSPs, J. Comput. System Sci. 67 (2003) 212-243.

[7] N. Alon, E. Fischer, I. Newman and A. Shapira: A Combinatorial Characterization of the Testable Graph Properties: It's All About Regularity, Proc. of the $38^{\text {th }}$ ACM Symp. Theor. of Comp. (STOC) (2006) 251-260.

[8] N. Alon and A. Shapira: A Characterization of the (natural) Graph Properties Testable with One-Sided Error, SIAM J. Computing 37 (2008), 1703-1727.

[9] N. Alon and U. Stav: What is the furthest graph from a hereditary property? Random Struc. Alg. 33 (2008), 87-104.

[10] N. Alon, A. Naor: Approximating the Cut-Norm via Grothendieck's Inequality SIAM J. Computing 35 (2006), 787-803.

[11] O. Angel and B. Szegedy (unpublished)

[12] S. Arora, D. Karger and M. Karpinski: Polynomial time approximation schemes for dense instances of NP-hard problems, Proc. 27-th ACM STOC (1995), 284-293.

[13] A.-L. Barabási: Linked: The New Science of Networks, Perseus, Cambridge, MA (2002).

[14] I. Benjamini, L. Lovász: Global Information from Local Observation, Proc. 43rd Ann. Symp. on Found. of Comp. Sci. (2002), 701-710.

[15] I. Benjamini, G. Kozma, L. Lovász, D. Romik, G. Tardos: Waiting for a bat to fly by (in polynomial time), Combinatorics, Probability and Computing 15 (2006), 673-683.

[16] I. Benjamini and O. Schramm: Recurrence of Distributional Limits of Finite Planar Graphs, Electronic J. Probab. 6 (2001), paper no. 23, 1-13.

[17] I. Benjamini, O. Schramm, A. Shapira: Every Minor-Closed Property of Sparse Graphs is Testable, http://front.math.ucdavis.edu/0801.2797 Proc. 40-th STOC (2008), 393-402.

[18] G.R. Blakley and P.A. Roy: A Hölder type inequality for symmetric matrices with nonnegative entries, Proc. Amer. Math. Soc. 16 (1965) 1244-1245.

[19] B. Bollobás: Relations between sets of complete subgraphs, in: Combinatorics, Proc. 5th British Comb. Conf. (ed. C.St.J.A. Nash-Williams, J. Sheehan), Utilitas Math. (1975), 79-84. 
[20] B. Bollobás: Random Graphs, Second Edition, Cambridge University Press, 2001.

[21] B. Bollobas, C. Borgs, J. Chayes, O. Riordan: Percolation on dense graph sequences, http://arxiv.org/abs/math/0701346

[22] B. Bollobas, S. Janson, O. Riordan: The phase transition in inhomogeneous random graphs, http://arxiv.org/abs/math/0701346 Random Struc. Alg. 31 (2007), 3-122.

[23] B. Bollobas, V. Nikiforov: An Abstract Regularity Lemma, http://arxiv.org/abs/ 0704.2450 in: Building Bridges (Grotschel, Katona eds.), Bolyai Soc. Math. Studies 19, Springer 2008, 219-240.

[24] B. Bollobas, O. Riordan: Sparse graphs: metrics and random models, http://arxiv. org/abs/0708.1919

[25] C. Borgs, J. Chayes, J. Kahn and L. Lovász: Left and right convergence of graphs with bounded degree (in preparation).

[26] C. Borgs, J. Chayes, L. Lovász: Moments of Two-Variable Functions and the Uniqueness of Graph Limits, http://www.cs.elte.hu/ lovasz/limitunique.pdf Geom. Func. Anal. (to appear).

[27] C. Borgs, J. Chayes, L. Lovász, V.T. Sós, K. Vesztergombi: Counting graph homomorphisms, in: Topics in Discrete Mathematics (ed. M. Klazar, J. Kratochvil, M. Loebl, J. Matoušek, R. Thomas, P. Valtr), Springer (2006), 315-371.

[28] C. Borgs, J.T. Chayes, L. Lovász, V.T. Sós, B. Szegedy and K. Vesztergombi: Graph Limits and Parameter Testing, Proc. 38th Annual ACM Symp. on Theory of Computing (2006), 261-270.

[29] C. Borgs, J.T. Chayes, L. Lovász, V.T. Sós, and K. Vesztergombi: Convergent Graph Sequences I: Subgraph frequencies, metric properties, and testing, Advances in Math. 219 (2008), 1801-1851.

[30] C. Borgs, J.T. Chayes, L. Lovász, V.T. Sós, and K. Vesztergombi: Convergent Graph Sequences II: Multiway Cuts and Statistical Physics (submitted), http://www.cs.elte.hu/ lovasz/ConvRight.pdf

[31] C. Borgs, J.T. Chayes, L. Lovász, V.T. Sós, and K. Vesztergombi: Limits of randomly grown graph sequences (submitted), http://arxiv.org/abs/0905.3806.

[32] M.-D. Choi: Tricks or Treats with the Hilbert Matrix, Amer. Math. Monthly 90 (1983), 301-312.

[33] F. Chung, R.L. Graham and R.M. Wilson: Quasi-random graphs, Combinatorica 9 (1989), 345-362.

[34] F. Chung, R.L. Graham: Quasi-Random Hypergraphs, Proc. Nat. AcadŚci. 86 (1989), pp. 8175-8177.

[35] P. Diaconis and S. Janson: Graph limits and exchangeable random graphs, http://eprintweb.org/S/authors/math/ja/Janson/3

[36] L. Devroye: Branching processes and their applications in the analysis of tree structures and tree algorithms", in: Probabilistic Methods for Algorithmic Discrete Mathematics, ed. M. Habib, C. McDiarmid, J. Ramirez-Alfonsin and B. Reed, pp. 249-314, Springer-Verlag, Berlin, 1998.

[37] G. Elek: On limits of finite graphs, Combinatorica 27 (2007), 503-507.

[38] G. Elek: A Regularity Lemma for Bounded Degree Graphs and Its Applications: Parameter Testing and Infinite Volume Limits, http://arxiv.org/abs/0711. 2800

[39] G. Elek: The Strong Approximation Conjecture holds for amenable groups, http://arxiv.org/abs/math/0511655.

[40] G. Elek: The combinatorial cost, http://arxiv.org/PS_cache/math/pdf/0608/ 0608474v1.pdf

[41] G. Elek and G. Lippner: An analogue of the Szemeredi Regularity Lemma for bounded degree graphs, http://arxiv.org/abs/0809.2879

[42] G. Elek, B. Szegedy: Limits of Hypergraphs, Removal and Regularity Lemmas. A Non-standard Approach, http://arxiv.org/0705.2179 
[43] P. Erdös, L. Lovász, J. Spencer: Strong independence of graphcopy functions, in: Graph Theory and Related Topics, Academic Press, 165-172.

[44] P. Erdös, A. Rényi: On random graphs I, Publ. Math. Debrecen 6 (1959), 290-297.

[45] E. Fischer: The art of uninformed decisions: A primer to property testing, The Computational Complexity Column of the Bulletin of the European Association for Theoretical Computer Science 75 (2001), 97-126.

[46] E. Fischer, I. Newman: Testing versus Estimation of Graph Properties, Proc. 37-th ACM STOC (2005), 138-146.

[47] D.C. Fisher: Lower bounds on the number of triangles in a graph, J. Graph Theory 13 (1989), 505-512.

[48] D.C. Fisher and J. Ryan: Conjectures on the number of complete subgraphs, in: Proc. of the 20-th Southeastern Conf. on Comb., Graph Theory, and Computing, Congr. Numer. 70 (1990), 217-219.

[49] D.C. Fisher and A. Solow: Dependence polynomials, Discrete Math. 82 (1990), 251-258.

[50] P. Frankl and J. Pach: An extremal problem on $K_{r}$-free graphs, J. Graph Theory 12 (1988), 519-523.

[51] M. Freedman, L. Lovász, A. Schrijver: Reflection positivity, rank connectivity, and homomorphisms of graphs, J. Amer. Math. Soc. 20 (2007), 37-51.

[52] A. Frieze and R. Kannan: Quick approximation to matrices and applications, Combinatorica 19, 175-220.

[53] D. Gaboriau: Invariants $l^{2}$ de relations dequivalence et de groupes, Publ. Math. Inst. Hautes. Ètudes Sci. 95 (2002), 93-150.

[54] S. Gerke, A. Steger: The sparse regularity lemma and its applications, Surveys in Combinatorics (2005), 227-258.

[55] E.N. Gilbert: Random graphs, Ann. Math. Stat. 30 (1959), 1141-1144.

[56] O. Goldreich, S. Goldwasser and D. Ron: Property testing and its connection to learning and approximation, J. ACM 45 (1998), 653-750.

[57] O. Goldreich and L. Trevisan: Three theorems regarding testing graph properties, Random Structures and Algorithms, 23 (2003), 23-57.

[58] A.W. Goodman: On sets of aquaintences and strangers at any party, Amer. Math. Monthly 66 (1959) 778-783.

[59] W.T. Gowers: Lower bounds of tower type for Szemerédi's Uniformity Lemma, Geom. Func. Anal. 7 (1997), 322-337.

[60] W.T. Gowers: Quasirandomness, counting and regularity for 3-uniform hypergraphs, Combin. Probab. Comput. 15 (2006), 143-184.

[61] W.T. Gowers: Hypergraph regularity and the multidimensional Szemeredi theorem, Annals of Math. 166 (2007), 897-946.

[62] M. Gromov: Metric structures for Riemannian and non-Riemannian spaces, Birkhäuser (1999).

[63] E. Györi, J. Pach, M. Simonovits: On the maximal number of certain subgraphs in $K_{r}$-free graphs, Graphs and Combin. 7 (1991), 31-37.

[64] H. Hatami: Graph norms and Sidorenko's conjecture, http://arxiv.org/abs/ 0806.0047 Israel J. Math. (to appear).

[65] J. Haviland, A. Thomason: Pseudo-random hypergraphs. Graph theory and combinatorics (Cambridge, 1988). Discrete Math. 75 (1989), 255-278.

[66] J. Haviland, A. Thomason: On testing the "pseudo-randomness" of a hypergraph. Discrete Math. 103 (1992), 321-327.

[67] P. Hell and J. Nešetřil: Graphs and Homomorphisms, Oxford University Press, 2004.

[68] S. Janson, T. Luczak and A. Ruczynski: Random Graphs, Wiley, 2000.

[69] A. Kechris and B.D. Miller: Topics in orbit equivalence theory, Lecture Notes in Mathematics 1852. Springer-Verlag, Berlin, 2004. 
[70] J. Kock: Frobenius Algebras and 2D Topological Quantum Field Theories, London Math. Soc. student texts, Cambridge University Press (2003).

[71] Y. Kohayakawa: Szemerédi's regularity lemma for sparse graphs, in: Sel. Papers Conf. Found. of Comp. Math., Springer (1997), 216-230.

[72] Y. Kohayakawa, V. Rödl: Szemerd́i's regularity lemma and quasi-randomness, in: Recent Advances in Algorithms and Combinatorics, CMS Books Math./Ouvrages Math. SMC 11, Springer, New York (2003), 289-351.

[73] J. Komlós and M. Simonovits: Szemerédi's Regularity Lemma and its applications in graph theory, in: Combinatorics, Paul Erdos is Eighty (D. Miklos et. al, eds.), Bolyai Society Mathematical Studies 2 (1996), pp. 295-352.

[74] G. Kun (personal communication).

[75] L. Lovász: Operations with structures, Acta Math. Hung. 18, 321-328.

[76] L. Lovász: Direct product in locally finite categories, Acta Sci. Math. Szeged 23, 319-322.

[77] L. Lovász: Connection matrices, in: Combinatorics, Complexity and Chance, A Tribute to Dominic Welsh Oxford Univ. Press (2007), 179-190.

[78] L. Lovász: The rank of connection matrices and the dimension of graph algebras, Eur. J. Comb. 27 (2006), 962-970.

[79] L. Lovász: Discrete Analytic Functions: An Exposition, in: Surveys in Differential Geometry IX, Eigenvalues of Laplacians and other geometric operators (Ed. Grigor'yan A., Yau S.-T.), Int. Press, Somerville, MA (2004), 241-273.

[80] L. Lovász, A. Schrijver: Graph parameters and semigroup functions, European Journal of Combinatorics (2007), doi:10.1016/j.ejc.2007.11.008

[81] L. Lovász, A. Schrijver: Dual graph homomorphisms, J. Comb. Theory (A) (to appear).

[82] L. Lovász, A. Schrijver: Semidefinite functions on categories, Elec. J. Comb. 16 (2) (2009-2010), No. R14.

[83] L. Lovász, M. Simonovits: On the number of complete subgraphs of a graph II, in: Studies in Pure Math., To the memory of P. Turán (ed. P. Erdös), Akadémiai Kiadó, 459-495.

[84] L. Lovász, V.T. Sós: Generalized quasirandom graphs, J. Comb. Th. B 98 (2008), $146-163$.

[85] L. Lovász, B. Szegedy: Limits of dense graph sequences, J. Comb. Theory B 96 (2006), 933-957.

[86] L. Lovász, B. Szegedy: Contractors and connectors in graph algebras, J. Comb. Th. B J. Graph Theory 60 (2009), 11-31.

[87] L. Lovász and B. Szegedy: Szemerédi's Lemma for the analyst, Geom. Func. Anal. 17 (2007), 252-270.

[88] L. Lovász, B. Szegedy: Testing properties of graphs and functions, to appear in Isr. J. Math, ftp://ftp.research.microsoft.com/pub/tr/TR-2005-110.pdf

[89] L. Lovász and B. Szegedy: The graph theoretic moment problem (manuscript) http://www.cs.elte.hu/ lovasz/moment.pdf

[90] L. Lovász and B. Szegedy: Finitely forcible graphons (manuscript) http://arxiv.org/ abs/0901.0929

[91] L. Lovász and B. Szegedy: Random Graphons and a Weak Positivstellensatz for Graphs (manuscript) http://arxiv.org/abs/0902.1327

[92] R. Lyons: Asymptotic enumeration of spanning trees Combin. Prob. Comput. 14 (2005) 491-522.

[93] J. Matoušek: Using the Borsuk-Ulam Theorem: Lectures on Topological Methods in Combinatorics and Geometry Springer, 2003.

[94] J.W. Moon, L. Moser: Mat. Kut. Int. Közl. 7 (1962), 283-286.

[95] V. Nikiforov: The number of cliques in graphs of given order and size, http: //arxiv.org/abs/0710.2305 
[96] O. Pikhurko: An Analytic Approach to Stability, http://arxiv.org/abs/0812.0214

[97] A. Pultr: Isomorphism types of objects in categories determined by numbers of morphisms, Acta Sci. Math. Szeged 35 (1973), 155-160.

[98] A.A. Razborov: Flag Algebras, Journal of Symbolic Logic, 72 (2007), 1239-1282.

[99] A.A. Razborov: On the minimal density of triangles in graphs, Combinatorics, Probability and Computing 17 (2008), 603-618.

[100] V. Rödl, J. Skokan: Regularity lemma for $k$-uniform hypergraphs, Random Structures Algorithms 25 (2004), 1-42.

[101] R. Rubinfeld and M. Sudan: Robust characterization of polynomials with applications to program testing, SIAM J. on Computing 25 (1996), 252-271.

[102] O. Schramm: Hyperfinite graph limits, http://arxiv.org/PS_cache/arxiv/pdf/0711/ 0711.3808v1.pdf

[103] A. Schrijver: Graph invariants in the edge model, in: Building Bridges - Between Mathematics and Computer Science (M. Grötschel, G.O.H. Katona, eds.), Springer, Berlin, 2008, pp. 487-498.

[104] A. Schrijver, Polynomial and tensor invariants and combinatorial parameters, http://homepages.cwi.nl/ lex/files/tensorc_long.pdf

[105] A. Schrijver, Tensor subalgebras and first fundamental theorems in invariant theory, Journal of Algebra 319 (2008) 1305-1319.

[106] A. Schrijver, Graph invariants in the spin model, http://homepages.cwi.nl/ lex/ files/grvm.pdf

[107] A. Sidorenko: A correlation inequality for bipartite graphs, Graphs and Combin. 9 (1993), 201-204.

[108] M. Simonovits, V.T. Sós: Hereditary extended properties, quasi-random graphs and induced subgraphs, Combinatorics, Probability and Computing 12 (2003), 319-344.

[109] M. Simonovits, V.T. Sós: Hereditarily extended properties, quasi-random graphs and not necessarily induced subgraphs. Combinatorica 17 (1997), 577-596.

[110] B. Szegedy: Edge coloring models and reflection positivity, J. Amer. Math. Soc. 20 (2007), 969-988.

[111] B. Szegedy (private communication).

[112] E. Szemerédi: "On sets of integers containing no $k$ elements in arithmetic progression", Acta Arithmetica 27 (1975) 199-245.

[113] E. Szemerédi: Regular partitions of graphs, Colloque Inter. CNRS (J.-C. Bermond, J.-C. Fournier, M. Las Vergnas and D. Sotteau, eds.) (1978) 399-401.

[114] T. Tao: A variant of the hypergraph removal lemma, J. of Comb. Theory, Series A 113, 1257-1280.

[115] T.C. Tao: Szemerédis regularity lemma revisited, Contrib. Discrete Math. 1 (2006), $8-28$.

[116] T.C. Tao: The dichotomy between structure and randomness, arithmetic progressions, and the primes, in: Proc. Intern. Congress of Math. I, Eur. Math. Soc., Zrich, 2006.

[117] A. Thomason: Pseudorandom graphs, in: Random graphs '85 North-Holland Math. Stud. 144, North-Holland, Amsterdam, 1987, 307-331.

[118] W.T. Tutte: On the Birkhoff-Lewis equations, Discrete Math. 92 (1991), 417-425.

[119] W.T. Tutte: On the matrix of chromatic joins, J. Comb. Theory B 57 (1993), 269-288.

[120] H. Whitney: The coloring of graphs, Ann. of Math. 33 (1932), 688-718. 